ARTICLE

https://doi.org/10.1038/s41467-019-11071-6

\title{
SHB1 and CCA1 interaction desensitizes light responses and enhances thermomorphogenesis
}

Qingbin Sun (1D ${ }^{1}$, Shulei Wang (1D ${ }^{1}$, Gang $\mathrm{Xu}^{1}$, Xiaojun $\mathrm{Kang}^{2}$, Min Zhang (1] ${ }^{1} \& \mathrm{Min} \mathrm{Ni}{ }^{2}{ }^{2}$

Light and temperature are two important environmental signals to plants. After dawn, photoactivated phytochromes translocate into the nucleus and interact with a family of negative basic helix-loop-helix PIF regulators. Subsequent phosphorylation and degradation of PIFs triggers a series of photomorphogenic responses. However, excess light can damage the photosynthetic apparatus and leads to photoinhibition. Plants acclimate to a balanced state of photomorphogenesis to avoid photodamage. Here, we show that upregulation of PIF4 expression by SHB1 and CCA1 under red light represents a desensitization step. After dawn, the highly expressed circadian clock protein CCA1 brings circadian signals to the regulatory region of the PIF4 signaling hub. Recruitment of SHB1 by CCA1 modulates red light-specific induction of PIF4 expression thus integrating circadian and light signals. As noon approaches and light intensity and ambient temperature tend to increase, the SHB1-CCA1 interaction sustains PIF4 expression to trigger thermomorphogenic responses to changing light and temperature conditions.

\footnotetext{
${ }^{1}$ National Key Laboratory of Crop biology, College of life Sciences, Shandong Agricultural University, Taian 271018, China. ${ }^{2}$ Department of Plant and Microbial Biology, University of Minnesota at Twin Cities, Saint Paul, Minnesota 55108, USA. Correspondence and requests for materials should be addressed to M.N. (email: nixxx008@umn.edu)
} 
ight signal is an important abiotic environmental factor. The regulation of plant growth and development by light signals involves three major classes of photoreceptors, the red (R) and far-red (FR) light-absorbing phytochromes and the UV-A/ blue light-absorbing cryptochromes and phototropins ${ }^{1}$. Arabidopsis thaliana has five phytochrome genes, $P H Y A$ to $P H Y E^{2-4}$. Phytochromes (phy) are photo-reversible between the red absorbing form (Pr) and the far-red absorbing form (Pfr). Following conversion to the biologically active Pfr form, phytochromes translocate into the nucleus and interact with an important subfamily of bHLH transcription factors, the phytochrome interacting factors (PIFs). Their interaction leads to their phosphorylation, ubiquitination, and degradation via the $26 \mathrm{~S}$ proteasome and alters gene expression rapidly ${ }^{5-8}$. PIFs play a variety of roles in regulating plant light responses, such as seed germination, seedling skotomorphogenesis, de-etiolation, shade avoidance, and flowering $9-11$.

Photoreceptors and the circadian clock sense and integrate diurnal and seasonal changes in environmental signals and modulate plant growth and development. The circadian clock regulates adaptation of plants to the alternation of day and night. The initial model of the plant circadian clock is a feedback loop, including the central oscillator components circadian clock associated 1 (CCA1, an MYB transcription factor), late elongated hypocotyl (LHY, a homolog of CCA1), and timing of $\mathrm{CAB} 2$ expression 1 (TOC1) and its related pseudoresponse regulators (PRRs). CCA1 and LHY are the first two plant clock genes identified, and bind directly to the TOC1 promoter and repress TOC1 expression ${ }^{12-15}$. TOC1 is a DNAbinding transcriptional factor and functions as a general transcriptional repressor of clock genes including CCA1 and LHY ${ }^{16-18}$. Recently, a number of new components have been integrated into clock models. These include the reveille (RVE) family of MYB transcription factors, which act in a feedback loop as transcriptional activators ${ }^{19,20}$. In addition, lightregulated WD 1/2 (LWD1/2) and night light-inducible and clock-regulated $1 / 2(\mathrm{LNK} 1 / 2)$ are also transcriptional activators involved in circadian clock function ${ }^{21,22}$.

Light and temperature are the most dynamic parameters in plant growth and development. PIFs act as pivotal components in a cellular signaling hub integrating biotic and abiotic pathways to regulate plant growth ${ }^{10}$. For example, PIF4 mediates plant adaptation to elevated ambient temperature or thermomorphogenesis ${ }^{23,24}$. Developmental and morphological changes are induced by high ambient temperature below the heat stress range ${ }^{25}$. In Arabidopsis, the changes include increased elongation of hypocotyls and petioles, hyponastic growth, and development of thinner leaves ${ }^{26}$. In addition, pif 4 exhibits an early flowering phenotype compared with wild type under high ambient temperature ${ }^{23}$. PIF4 coordinates this response by activating hormonal modules that subsequently regulate growth. PIF4 interacts with brassinazole-resistant 1 (BZR1), a transcription factor induced by brassinosteroid, and activates the expression of a number of downstream genes that drive shoot organ elongation $^{27,28}$. PIF4 also directly regulates auxin levels by activating several auxin biosynthesis genes such as yucca 8 (YUC8) to promote hypocotyl elongation and hyponastic leaf growth ${ }^{29,30}$. TOC1, the evening-expressed circadian clock protein, directly interacts with PIF4 and prevents its activation of downstream target genes, thereby suppressing thermomorphogenesis specifically at the end of day and evening 24 .

Although light signals are fundamental to the growth and development of plants, excess light energy damages the photosynthetic apparatus and frequently enforces an inhibitory effect on photosynthesis known as photoinhibition ${ }^{31}$. Light inevitably generates excess reactive oxygen species (ROS) can be generated during photosynthesis under strong light, which can lead to photoinhibition and oxidative damage to the photosynthetic apparatus ${ }^{32-35}$. Photosynthetic organisms are able to acclimate to different environmental conditions to alleviate the detrimental effects of excess light on growth and viability ${ }^{36}$. For example, under excess light conditions, plants may acquire a balanced state of photomorphogenesis to avoid the absorption of excess light energy and to reduce photodamage through a feedback mechanism. Although the molecular mechanisms stimulating response to light are well established, those required for desensitization of perceived light signals remain less understood.

SHB1 was initially isolated from the gain-of-function mutant short hypocotyl under blue 1 Dominant (shb1-D) based on its long hypocotyl phenotype under red, far-red, and blue light ${ }^{37}$. In shb1$D$, a T-DNA is inserted 129 base pairs upstream of the $S H B 1$ start codon and causes SHB1 overexpression. SHB1 contains an $\mathrm{N}$ terminal SPX domain and a C-terminal EXS domain homologous to yeast suppressor of yeast GPA1 (SYG1) family proteins ${ }^{37}$. Its N terminus retains the function of full-length SHB1, and overaccumulation of the SHB1 C terminus causes a dominantnegative phenotype ${ }^{38}$. PIF4 expression is increased in $\operatorname{shbl-D}$ compared with wild type and decreased in shb1 partial loss-offunction mutant specifically under red light ${ }^{37}$. The molecular mechanism by which SHB1 regulates PIF4 expression specifically under red light and its biological implication are still unknown. In this study, SHB1 hijacks the highly expressed central oscillator component CCA1 and LHY in the morning and is targeted to the PIF4 promoter. During the day when light intensity and temperature increase, the SHB1-CCA1/LHY interaction sustains PIF4 expression in response to both red light and higher ambient temperature. This mechanism serves two important purposes: upregulate PIF4 expression to desensitize light responses for optimal photomorphogenesis, and enhance plant thermomorphogenesis for better survival under elevated ambient temperature.

\section{Results}

SHB1, CCA1, and LHY regulate PIF4 expression under red light. In an early study, SHB1 specifically upregulated PIF4 expression under red light as assessed by real-time quantitative PCR and RNA gel blot hybridization analysis ${ }^{37}$. In general, PIF4 expression was induced by red light, downregulated in $\operatorname{shb} 1$ and upregulated in shb1-D compared with wild type under red light (Fig. 1a). Although PIF1, PIF3, PIF5, and PIF7 expression was induced by red light, only the red light-induced expression of PIF7 was partially compromised in shb1 but not in shb1-D (Supplementary Fig. 1a). The rhythmic expression of PIF4 is controlled by the circadian clock $^{39-41}$. We also examined the rhythmic expression of PIF4 in Col, shb1, Ws, and shb1-D seedlings under continuous red light after growth under 12-hr dark and 12-hr $2 \mu \mathrm{mol} \mathrm{m}^{-2} \mathrm{~s}^{-1}$ red light for 7 days (Fig. 1b, c). Samples were obtained at ZT0 and every $3 \mathrm{~h}$ thereafter for $36 \mathrm{~h}$. Both shb1 and shb1-D mutations affected the magnitude but not the rhythmic pattern of PIF4 expression.

When PIF4 was driven by its native promoter, PIF4 protein accumulation correlated with PIF4 transcription, and PIF4 protein accumulated during the light period from ZT0 to ZT8 but not in the dark period from ZT12 to ZT20 under short days $(8 \mathrm{~h} \text { white light } / 16 \mathrm{~h} \text { dark })^{42}$. Under a 12 -hr red light and 12 -hr dark photoperiod, PIF4 protein was detectable after ZT3 and reached its peak accumulation at ZT9, correlating well with PIF4 mRNA level (Fig. 1b-e). Before ZT3, the lower level of PIF4 accumulation was probably due to the low level of PIF4 mRNA and/or PIF4 degradation after the initial illumination with red 

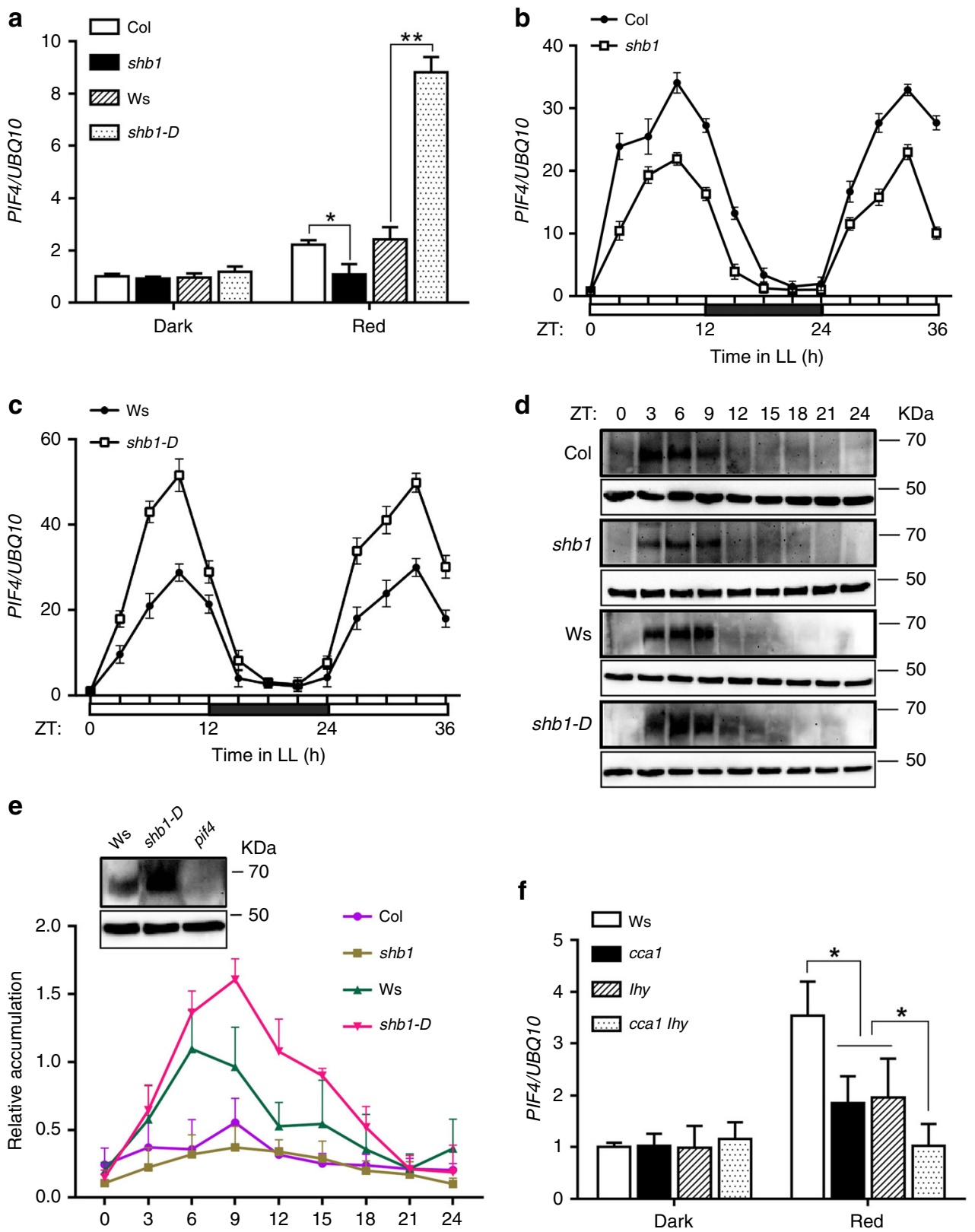

Fig. 1 SHB1 and CCA1/LHY regulate PIF4 expression under red light. a PIF4 expression in 4-day-old Col, shb1, Ws, and shb1-D seedlings in the dark and under $15 \mu \mathrm{mol} \mathrm{m} \mathrm{m}^{-2} \mathrm{~s}^{-1}$ red light for $3 \mathrm{hr} .{ }^{*}$ and ${ }^{\star \star}$ indicate significance levels $p<0.05$ and $p<0.01$, respectively. ns indicates not significant by Student's two-tailed heteroscedastic $t$ tests in this figure and subsequent figures. PIF4 expression in Col and shb1 b or Ws and shb1-D c under continuous red light after entrained under 12-hr light and 12-hr dark for 7 days from two biological replicates. d PIF4 protein accumulation in Col, shb1, Ws, and shb1-D under 12hr red light and 12-hr dark photoperiod, shown as representative images from three biological replicates. e PIF4 protein quantification normalized to actin with standard error bars. The control blot shows PIF4 accumulation in Ws, shb1-D and pif4 at ZT6. f PIF4 expression in Ws, ccal, Ihy, and ccal Ihy in the dark and under $15 \mu \mathrm{mol} \mathrm{m}{ }^{-2} \mathrm{~s}^{-1}$ red light. Expression of PIF4 in each sample was normalized to that of UBQ10, and data are presented as the means \pm SE. Source data are provided as a Source Data file

light. PIF4 accumulation was strongly enhanced in shb1-D from ZT6 to ZT12 but moderately reduced in shb1 from ZT6 to ZT12 and beyond (Fig. 1e).

SHB1 is unable to target to the PIF4 locus given that it contains no recognizable DNA-binding domain. Other transcription factors likely recruit SHB1 to the PIF4 promoter. We identified several potential MYB-binding elements ATATC(T/A) in the PIF4 promoter (http://meme-suite.org/). In Arabidopsis, MYB family transcription factors have multiple functions ${ }^{43}$. The two central circadian clock components, CCA1 and LHY, are potential candidates. PIF4 expression was reduced in the ccal or the lhy single mutant, and was further reduced in the ccal lhy double mutant (Fig. 1f). PIF4 expression was not altered in ccal or lhy single mutants or in the ccal lhy double mutant in the dark. The rhythmic expression of PIF4 is controlled by the circadian clock $^{39-41}$. In the ccal lhy double mutant, the rhythmic expression of PIF4 was completely compromised (Supplementary Fig. 1b). In addition, SHB1 expression was not rhythmically expressed compared with CCA1 and LHY (Supplementary Fig. 1c). Therefore, CCA1 and LHY are required for the rhythmic expression of PIF4, and SHB1 enhances but does not alter the pattern of PIF4 rhythmic expression. 
a
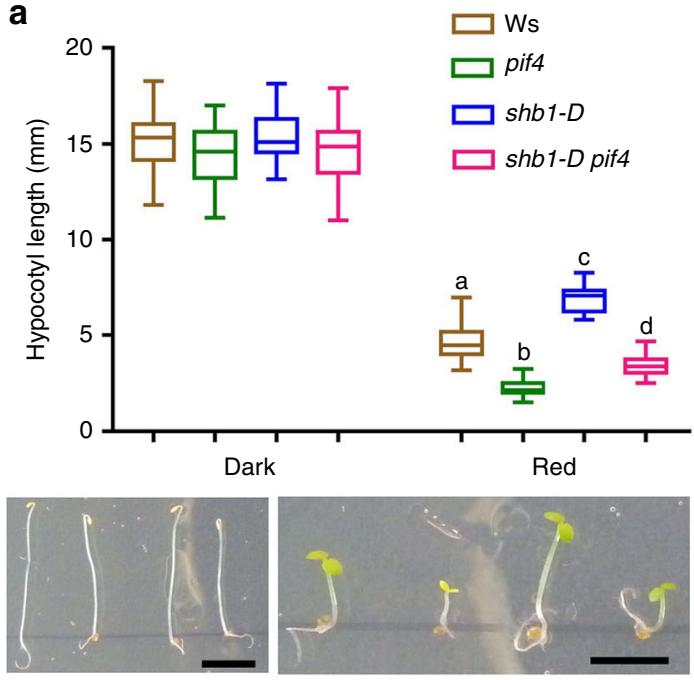

C

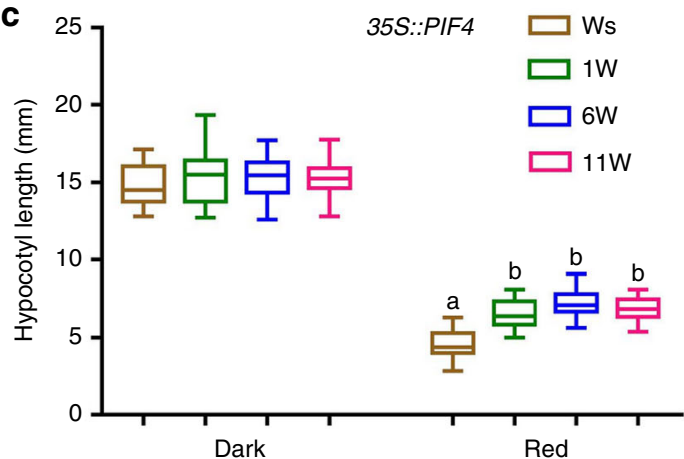

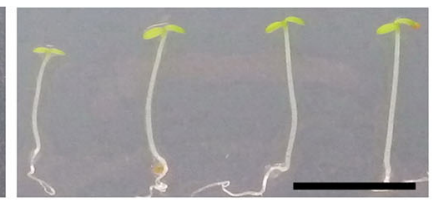

d

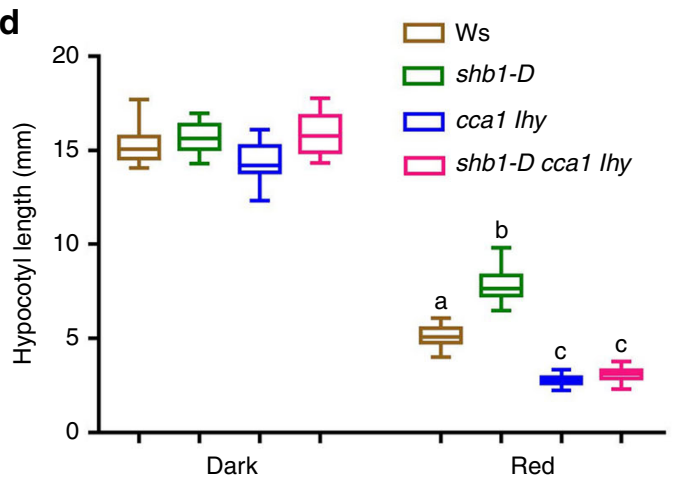

b
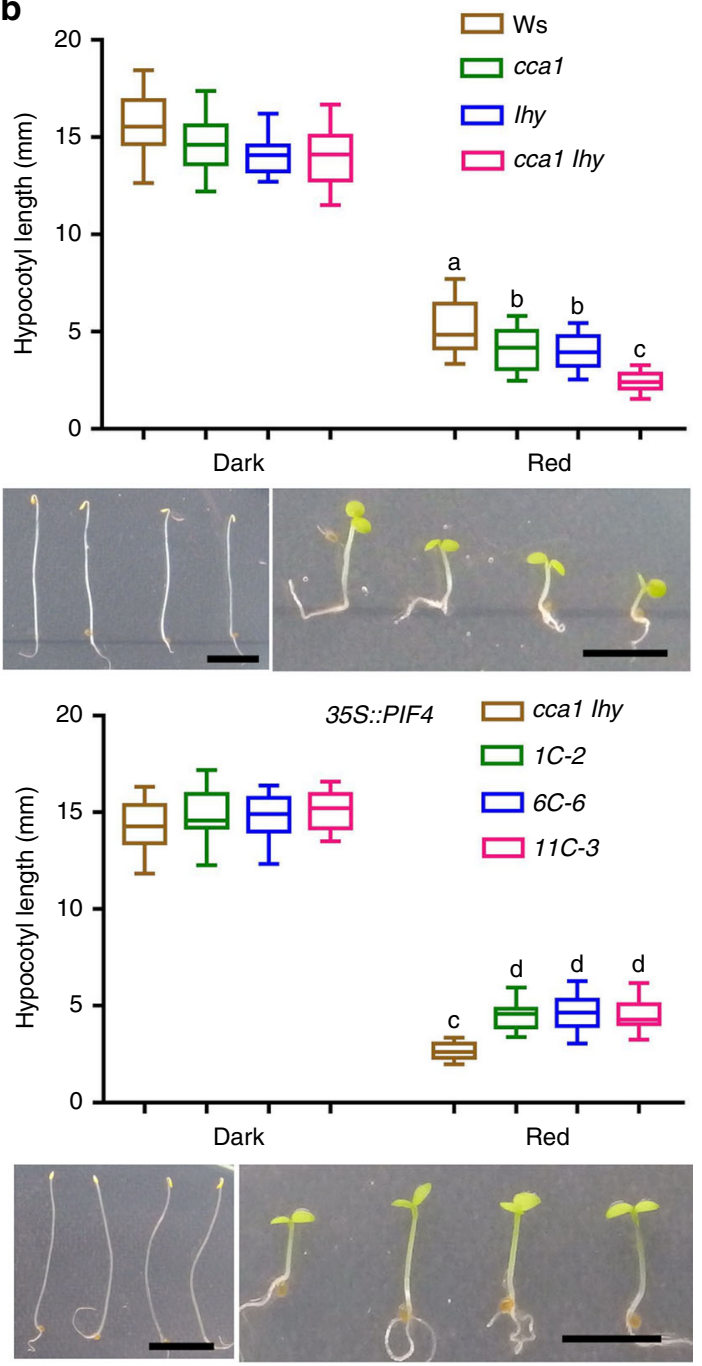

e

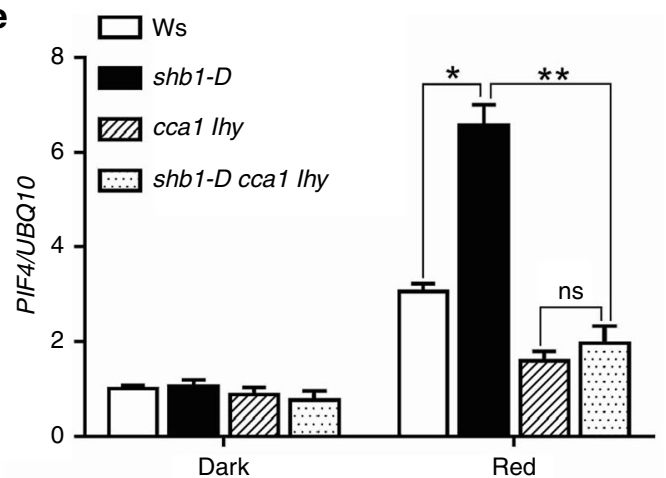

Fig. 2 Genetic interaction of PIF4 with SHB1 or CCA1/LHY. Hypocotyl lengths of Ws, pif4, shb1-D, and shb1-D pif4 a; Ws, ccal, Ihy, and cca1 Ihy $\mathbf{b}$; three 35 S:: PIF4:GFP (pMDC83) transgenic lines in Ws or ccal Ihy c; and Ws, shb1-D, ccal lhy, and shb1-D ccal lhy $\mathbf{d}$ in the dark or under $10 \mu \mathrm{mol} \mathrm{m}^{-2} \mathrm{~s}^{-1}$ red light for 4 days. Bar $=5 \mathrm{~mm}$. Box plots display medians as horizontal lines, interquartile ranges as boxes and whiskers extending 1.5 times the interquartile range in this and subsequent figures. Significance levels by Student's two-tailed heteroscedastic $t$ tests in a: $p<0.001$ between $\mathbf{a}$ and $\mathbf{b}$, $\mathbf{a}$ and $\mathbf{c}, \mathbf{a}$ and $\mathbf{d}, \mathbf{b}$ and $\mathbf{d}$, or $\mathbf{c}$ and $\mathbf{d}$; in $\mathbf{b}$ : $p<0.01$ between $\mathbf{a}$ and $\mathbf{b}, p<0.001$ between $\mathbf{a}$ and $\mathbf{c}$ or $\mathbf{b}$ and $\mathbf{c}$; in $\mathbf{c}: p<0.001$ between $\mathbf{a}$ and $\mathbf{b}$ or $\mathbf{c}$ and $\mathbf{d}$; in $\mathbf{d}: p<0.001$ between $\mathbf{a}$ and $\mathbf{b}$, a and $\mathbf{c}$ or $\mathbf{b}$ and $\mathbf{c}$. e PIF4 expression in Ws, shb1-D, cca1 Ihy, and shb1-D cca1 Ihy. PIF4 expression in each sample was normalized to that of UBQ10, and data are presented as the means \pm SE. Source data are provided as a Source Data file

Genetic interaction between PIF4 and SHB1, CCA1, and LHY. We investigated the genetic interaction between SHB1 and PIF4 by measuring the hypocotyl elongation of Ws, pif4, shb1-D and the shb1-D pif4 double mutant under red light. shb1-D exhibited a long hypocotyl, and pif4 exhibited a short hypocotyl compared with wild type. The shb1-D pif4 double mutant exhibited a slightly longer hypocotyl compared with pif4, but it was considerably much shorter than that of shb1-D (Fig. 2a). The shb1-D mutation still increased hypocotyl length in the pif4 mutant background or SHB1 also promoted hypocotyl elongation independent of PIF4, 
SHB1 may target to other genes in addition to PIF4 to influence hypocotyl elongation.

Although CCA1 and $L H Y$ are redundant genes, the ccal or lhy single mutant exhibited a short hypocotyl similar to that of pif4, and the ccal lhy double mutant exhibited a considerably shorter hypocotyl compared with each single ccal or lhy mutant (Fig. 2b). To study their epistasis, we overexpressed PIF4:GFP driven by the CaMV $35 \mathrm{~S}$ promoter in Ws and crossed three independent transgenes to the ccal lhy background (Supplementary Fig. 2a). The hypocotyl of the 35 S::PIF4:GFP transgenic lines was considerably elongated in Ws (Fig. 2c). Overexpression of PIF4::GFP in ccal lhy generated a shorter hypocotyl compared with that in Ws, but a considerably longer hypocotyl compared with the ccal lhy double mutant. Combining the results demonstrating that the red light-induced PIF4 expression relies on CCA1 and LHY (Fig. If and Supplementary Fig. 1b), we conclude that PIF4 acts downstream of CCA1 and LHY.

The shb1 mutation caused a minimal hypocotyl phenotype but affected PIF4 expression under red light ${ }^{37}$. The shb1 mutant allele also showed a partial loss-of-function phenotype in endosperm proliferation and cellularization ${ }^{44,45}$. Triple shb1 ccal lhy mutant was constructed, and the hypocotyl length and PIF4 expression were barely affected when shbl was introduced to ccal lhy (Supplementary Fig. 2b, c). In shb1, a T-DNA is inserted at the 8th intron of the SHB1 gene (SALK_128406), and a truncated message was still produced (Supplementary Fig. 2d, e). Given the extremely low level of SHB1 expression and the low sensitivity of Taq polymerase used for semi-quantitative PCR analysis, we previously failed to detect the truncated message ${ }^{37}$. The fulllength SHB1 protein contains 745 amino acids, and shb1 lacks the C-terminal 223 amino acids but retains the N-terminal 522 amino acids. Given that the $\mathrm{N}$ terminus of SHB1 is important for its function $^{38}$, the truncated SHB1 in $s h b 1$ is partially functional. In contrast, a shb1-D ccal lhy triple mutant showed a hypocotyl phenotype and PIF4 expression similar to that of the ccal lhy double mutant (Fig. 2d, e and Supplementary Fig. 2f). We examined the expression of SHB1 in shb1-D and shb1-D ccal lhy triple mutant (Supplementary Fig. 2g). The levels of SHB1 transcripts are comparable in shb1-D and shb1-D ccal lhy. Thus, the promotion of hypocotyl and PIF4 expression by SHB1 under red light critically relies on CCA1 and LHY.

Regulation of PIF4 expression enhances thermomorphogenesis. Given that PIF4 is required for thermomorphogenesis, the induction of PIF4 expression by SHB1 and CCA1 may affect plant thermomorphogenesis. We examined hypocotyl growth responses of Ws, shb1, pif4, shb1-D, and the shb1-D pif4 double mutant to warm temperatures. Col or Ws showed a clear thermos-response and this response was slightly reduced in $s h b 1$ but almost disappeared in pif4 (Fig. 3a). shb1-D enhanced the thermos-response with an elongated hypocotyl, and this enhancement was not completely blocked in shb1-D pif4 double mutant. Therefore, the thermo-induced hypocotyl elongation through PIF4 under higher temperature may not be entirely dependent on SHB1.

Furthermore, warm temperature-induced hypocotyl growth of either ccal or lhy was similar to that of Ws, and ccal lhy double mutations indeed reduced plant responses to warm temperatures (Fig. 3b). Overexpression of PIF4 driven by the $35 \mathrm{~S}$ promoter enhanced the thermo-response in either Ws wild type or ccal lhy backgrounds (Fig. $3 \mathrm{c}$ and Supplementary Fig. 3). PIF4overexpression plants grown at $20{ }^{\circ} \mathrm{C}$ exhibited a longer hypocotyl and increasing the temperature to $29^{\circ} \mathrm{C}$ caused a further elongation of the hypocotyl. However, the ratio of hypocotyl length at $20^{\circ} \mathrm{C}$ versus $29^{\circ} \mathrm{C}$ in PIF4-overexpression plants was comparable in either a wild type or ccal lhy double mutant background. Warm temperature-induced PIF4 expression was reduced in $\operatorname{shbl}$ and ccal lhy, but significantly enhanced in shb1-D compared with that in Col or Ws wild type (Fig. 3f).

Hypocotyls of the ccal lhy double mutant were elongated in response to high temperature, and PIF4 expression was still upregulated under high temperatures in ccal lhy. The degree of upregulation was comparable between the wild type and the mutants. Therefore, the induction of the PIF4-mediated thermosresponse under high temperature may not completely rely on CCA1 and LHY. In general, SHB1 and CCA1/LHY may not be required for thermos-activation of PIF4, but the overall increase in PIF4 transcription increases the magnitude of the thermoresponse. Both hypocotyl and PIF4 expression at $29^{\circ} \mathrm{C}$ in $\operatorname{shbl-D}$ ccal lhy triple mutant were higher than that of ccal lhy double mutant, but lower than that of shb1-D (Fig. 3d-f). In addition to CCA1/LHY, other transcription factors may participate in SHB1meditated thermos-responses.

SHB1, CCA1, and LHY associate with the PIF4 promoter. We assessed whether SHB1 directly regulates PIF4 expression although SHB1 does not contain a recognizable DNA-binding motif. We performed ChIP experiments with a SHB1:GFP transgene driven by the $35 \mathrm{~S}$ promoter and anti-GFP antibody followed by quantitative PCR (qPCR) analysis. We used four pairs of primers that detect different regions in the PIF4 promoter (Fig. 4a). The samples were collected at ZT3 (8 am as ZT0) for dark-grown plants without or with $3 \mathrm{~h}$ of red light treatment. SHB1 was associated with 4-3 fragment in the PIF4 promoter (Fig. 4b). Slight enrichment was observed for the 4-4 genomic fragment but the results were not statistically significant. The SHB1:GFP construct caused a longer hypocotyl phenotype and elevated PIF4 expression (Supplementary Fig. 4a, b).

Two MYB-binding sites, M1 and M2, were identified in these two fragments (Fig. 4a). MYB transcription factors CCA1 or LHY may mediate the association of between SHB1 and the PIF4 promoter. We performed ChIP experiments with CCA1:FLAG and LHY:MYC transgenes driven by the $35 \mathrm{~S}$ promoter and antiFLAG or anti-MYC antibodies, respectively. CCA1 and LHY were also associated with the PIF4 promoter, and the 4-3 fragment was enriched under red light (Fig. 4c and Supplementary Fig. 4c). We further examined whether SHB1 remained associated with the PIF4 promoter if CCA1 and LHY are mutated. SHB1 was unable to associate with the 4-3 genomic fragment in the PIF4 promoter in ccal lhy (Fig. 4c). We examined the accumulation of SHB1: GFP in Ws and ccal lhy, and their levels were comparable in either Ws or ccal lhy in the dark and under red light at the time points sampled for ChIP analysis (Supplementary Fig. 4d). SHB1: GFP overexpression caused a longer hypocotyl phenotype and elevated PIF4 expression under red light in Ws but not in ccal lhy (Supplementary Fig. 4e, f).

CCA1:FLAG and LHY:MYC did not associated with the PIF4 promoter in the dark (Fig. 4c and Supplementary Fig. 4c). CCA1: FLAG or LHY:MYC levels were comparable in the dark and under red light for $3 \mathrm{~h}$ when we sampled for ChIP analysis (Supplementary Fig. 4d). LUX, a component of the evening complex (EC), recognizes a consensus element GATWCG and two different types of degenerate elements, GATWYG or GATWCK $^{46}$. The 4-4 fragment has one consensus element GATTCG that is $210 \mathrm{bp}$ from the CCA1/LHY-binding element in the PIF4 promoter. The 4-3 fragment contains one degenerate element, GATTCC, which is 90 bp away from the CCA1/LHYbinding element. In addition, another degenerate element, GATTTG, is $1 \mathrm{bp}$ away from the forward primer of the 4-3 fragment. In the EC complex, LUX interacts with ELF3 and ELF3 
a

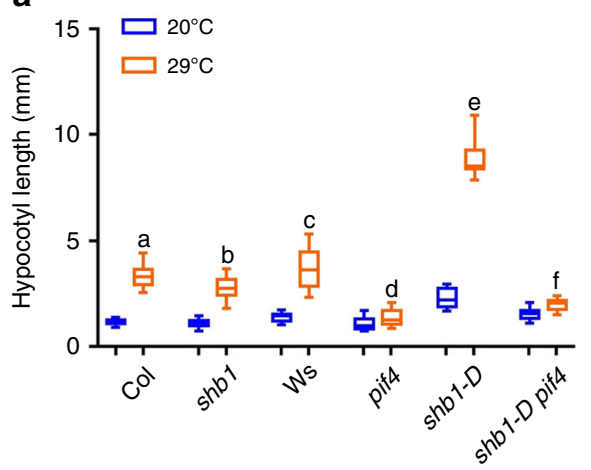

$20^{\circ} \mathrm{C}$

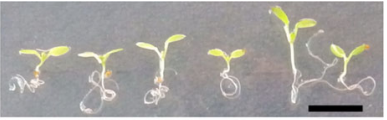

$29^{\circ} \mathrm{C}$

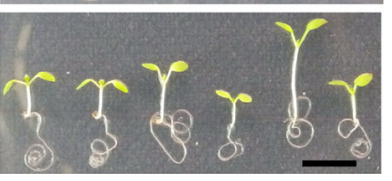

C

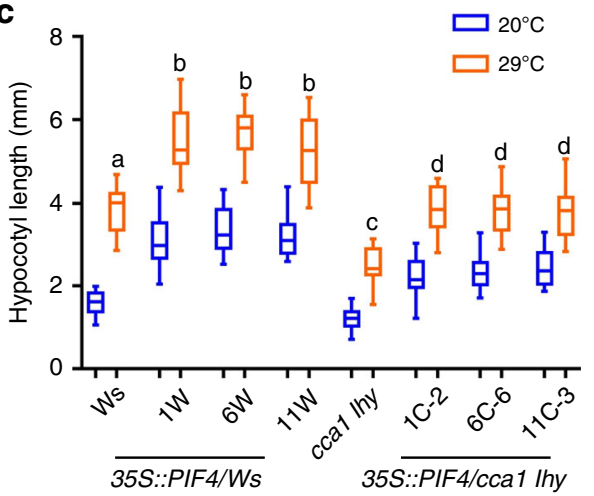

e

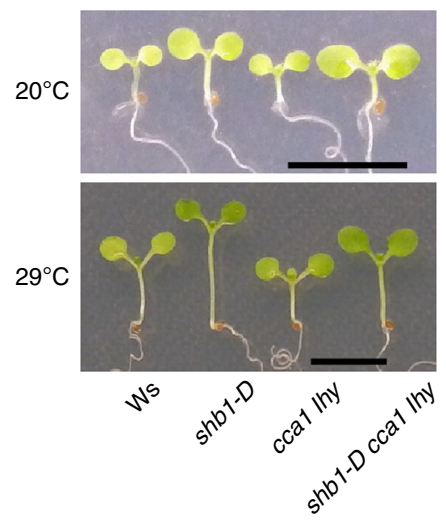

b
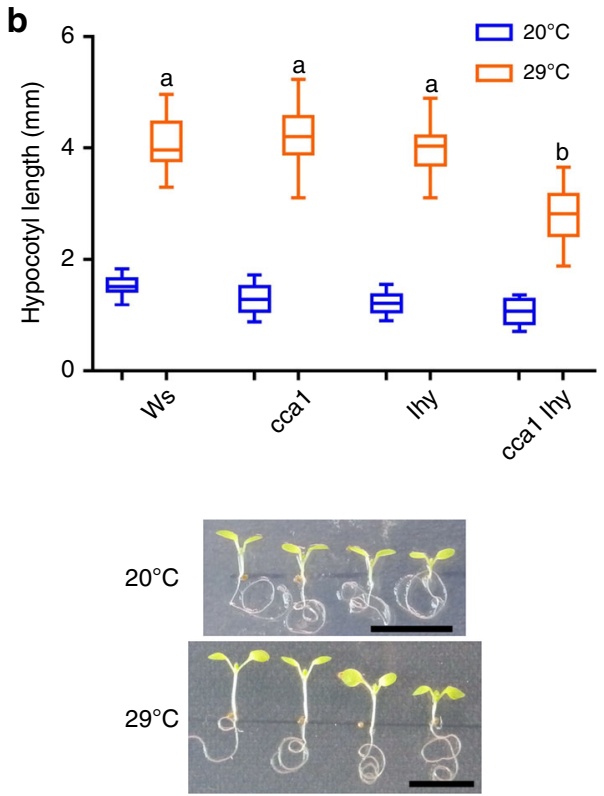

d

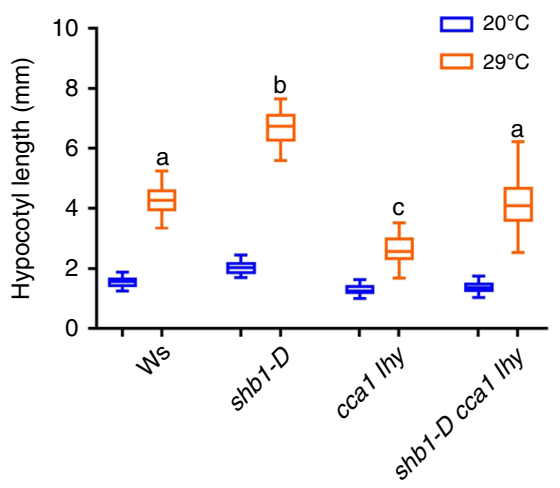

f

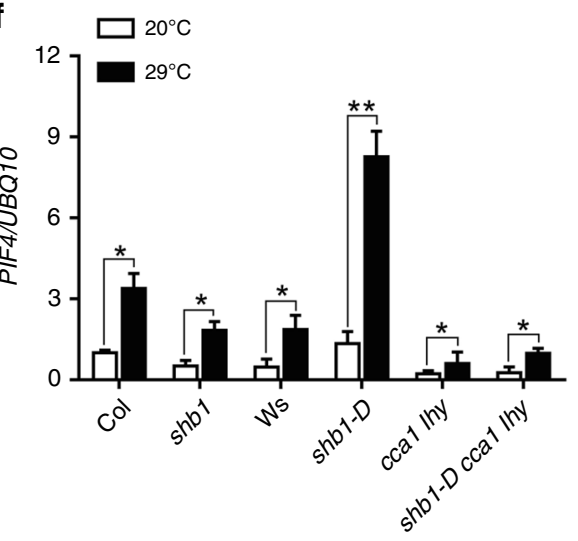

Fig. 3 CCA1/LHY and SHB1 enhance PIF4 expression and thermomorphogenesis. Hypocotyl lengths of Col, shb1, Ws, pif4, shb1-D, and shb1-D pif4 a; Ws, ccal, Ihy, and ccal Ihy $\mathbf{b}$; three 35 S:::PIF4:GFP lines in Ws or ccal Ihy $\mathbf{c}$; and Ws, shb1-D, ccal lhy, and shb1-D ccal Ihy d, e under $30 \mu \mathrm{mol} \mathrm{m}^{-2} \mathrm{~s}^{-1}$ white light at $20^{\circ} \mathrm{C}$ for 7 days or $20^{\circ} \mathrm{C}$ for 4 days followed by $29^{\circ} \mathrm{C}$ for 3 days. Bar $=5 \mathrm{~mm}$. Significance levels by Student's two-tailed heteroscedastic $t$ tests in a: $p<0.001$ between $\mathbf{a}$ and $\mathbf{b}, \mathbf{c}$ and $\mathbf{d}, \mathbf{c}$ and $\mathbf{e}, \mathbf{c}$ and $\mathbf{f}$, $\mathbf{d}$ and $\mathbf{e}$, or $\mathbf{e}$ and $\mathbf{f}$ and $p<0.01$ between $\mathbf{d}$ and $\mathbf{f}$; in $\mathbf{b}$ : $p<0.001$ between $\mathbf{a}$ and $\mathbf{b}$; in $\mathbf{c}$ : $p<0.001$ between $\mathbf{a}$ and $\mathbf{b}$ or $\mathbf{c}$ and $\mathbf{d}$; in $\mathbf{d}$ : $p<0.001$ between $\mathbf{a}$ and $\mathbf{b}$, $\mathbf{a}$ and $\mathbf{c}$ or $\mathbf{b}$ and $\mathbf{c}$. f PIF4 expression in Col, shb1, Ws, shb1-D, ccal Ihy, and shb1-D ccal Ihy. Seedlings were grown at $20^{\circ} \mathrm{C}$ for 5 days and then incubated at $20^{\circ} \mathrm{C}$ or $29^{\circ} \mathrm{C}$ for $4 \mathrm{~h}$. PIF4 expression in each sample was normalized to that of UBQ10, and data are presented as the means $\pm \mathrm{SE}$. Source data are provided as a Source Data file 


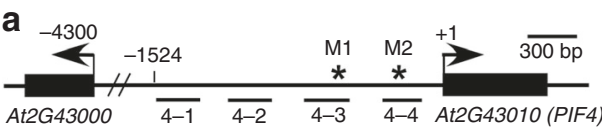

b

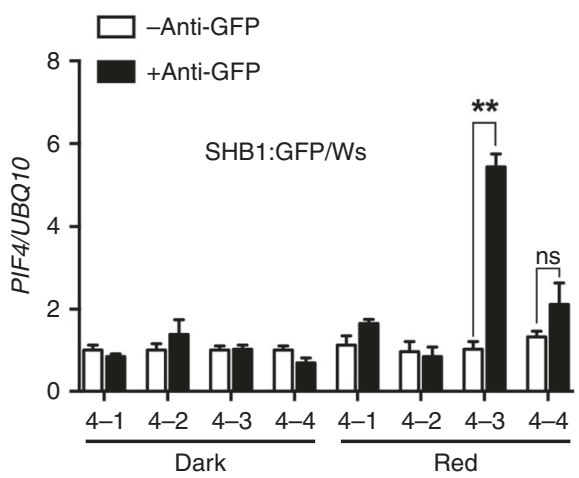

C
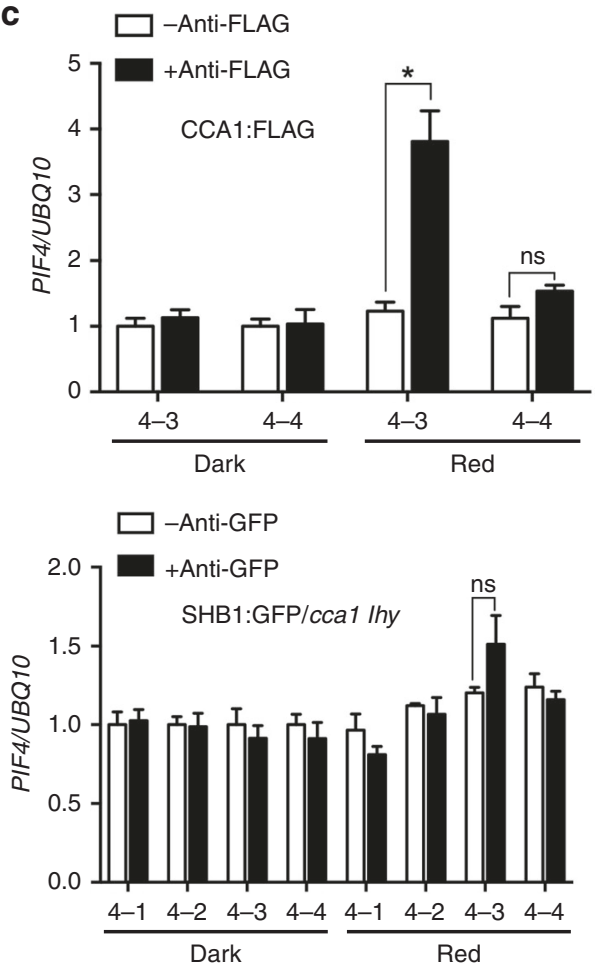

d
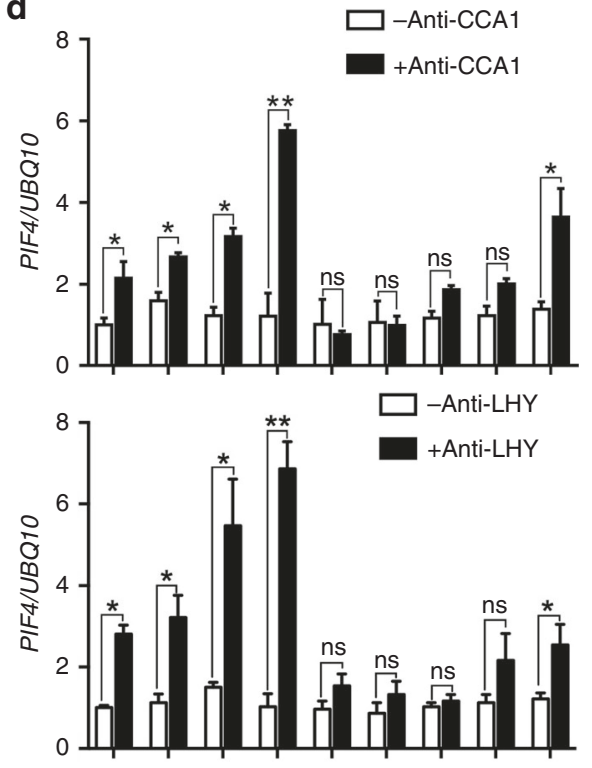

$\left.\begin{array}{c}10 \\ 8\end{array}\right] \quad \square$-Anti-GFP

SHB1:GFP

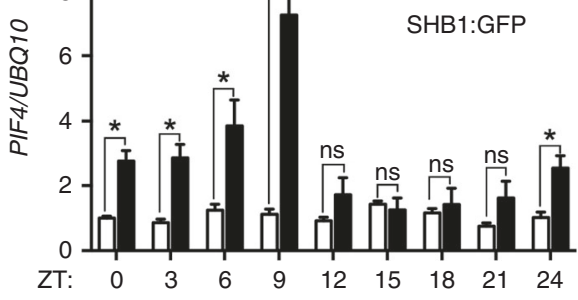

e

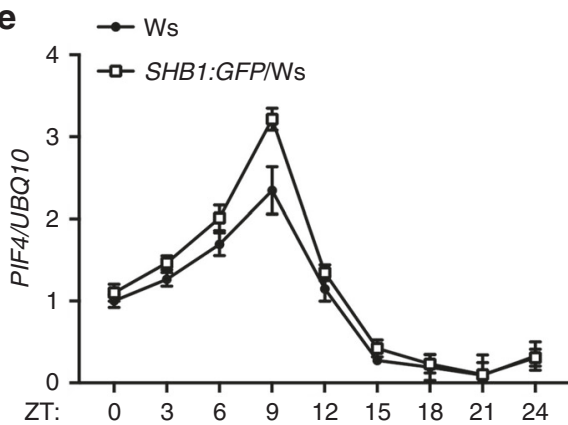

Fig. 4 SHB1 and CCA1/LHY are associated with the PIF4 promoter. a PIF4 promoter demonstrating the chromatin regions (4-1, 4-2, 4-3, and 4-4) used for ChIP-quantitative PCR analysis and the potential Myb-binding sites M1 and M2 (asterisks). Association of SHB1:GFP b and CCA1:FLAG or SHB1:GFP in cca1 Ihy c with the PIF4 promoter. d Rhythmic association of CCA1, LHY, or SHB1:GFP with the PIF4 promoter under 12-hr dark and 12-hr red light cycles from two biological replicates. Enrichment of DNA fragments was quantified by qPCR and normalized to that of UBQ10. e Rhythmic PIF4 expression at various ZT points in Ws and 35 S::SHB1:GFP transgenic plants under 12-hr dark and 12-hr red light cycles. Data are presented as the means \pm SE calculated from two biological replicates. Source data are provided as a Source Data file

interacts with ELF4. ELF4:GFP was associated with both 4-3 $(p<0.018)$ and $4-4 \quad(p<0.013)$ fragments in the dark, but less efficiently with the 4-4 $(p<0.045)$ fragment under red light (Supplementary Fig. 5a). ELF4:GFP accumulated at comparable levels in the dark and under red light (Supplementary Fig. 5b). Through ChIP-quantitative PCR assays and genome-wide expression profiling, PRR5, PRR7, and PRR9 also bind to the upstream regions of PIF4 and other key transcription factor genes, and repress their expression ${ }^{47}$. We hypothesize that the occupation of the PIF4 promoter by the entire EC complex and/ or the PRR proteins in the dark may interfere with the binding of CCA1 or LHY to the PIF4 promoter.
To correlate SHB1 binding to the PIF4 promoter with the regulation of PIF4 expression, we performed rhythmic ChIP assay for CCA1, LHY, and SHB1 (Fig. 4d). We used wild-type plants with antibodies against CCA1 or LHY and 35 S::SHB1: GFP transgenic plants. We used the $35 \mathrm{~S}$ promoter to drive SHB1 expression given that SHB1 native promoter is very weak and SHB1 expression is not rhythmic. The plants were grown under 12-hr red light and 12-hr dark and were sampled at ZT0 and every $3 \mathrm{~h}$ thereafter for $24 \mathrm{~h}$. CCA1, LHY, and SHB1 were associated with the PIF4 promoter from ZT0 to ZT9 but not thereafter (Fig. 4d). The ChIP peak at ZT9 coincided with a maximum level of PIF4 expression, and PIF4 mRNA levels 
declined after ZT9 (Fig. 4e). Surprisingly, the CCA1 ChIP peak did not coincide with the CCA1 mRNA peak (Supplementary Fig. 1c and Fig. 4d). There might be a gradual departure of the EC complex or PRRs. Alternatively, CCA1 and LHY proteins or the SHB1-CCA1/LHY complex is very stable under red light. As shown in an early study, a large number of CCA1 target genes of CCA exhibit a peak expression from ZT5 to ZT16 in LD diel conditions or LL constant light ${ }^{48}$. Among them, ERD7 has a similar expression pattern as PIF4. Therefore, CCA1 plays a potentially large role in the regulation of morningexpressed genes.

CCA1 and LHY bind the MYB element in the PIF4 promoter. We identified two MYB-binding elements, M1 and M2, in the PIF4 promoter (Fig. 5a and Supplementary Fig. 6a). In a yeast one-hybrid $(\mathrm{Y} 1 \mathrm{H})$ assay, CCAl and LHY bound to a DNA fragment that contains both M1 and M2 MYB binding elements in the PIF4 promoter (Fig. 5b). We then tested which MYBbinding element is recognized by CCA1 or LHY using a trimeric M1 or M2 element in tandem repeats (Fig. 5c and Supplementary Fig. 6b). CCA1 and LHY bound to the trimeric M1 element but not the trimeric M2 element. We subsequently mutated the M1 element or M2 element by changing two conserved bases and assessed whether CCA1 or LHY recognizes the mutated element (Fig. 5c and Supplementary Fig. 6b). Neither CCA1 nor LHY bound to the mutated trimeric M1 element.

PIF4 gene driven by a PIF4 promoter that bears either a wide type or a deleted M1 box was subsequently introduced into pif4 or shb1-D pif4 background (Fig. 5a). Three lines in each category with comparable transgene expression was examined for light- and thermo-responses (Supplementary Fig. 7a, b). The pPIF4::PIF4 cassette with a deleted M1 box hardly rescued the pif4 hypocotyl phenotype in either pif4 or shb1-D pif4 background compared with that with a wild-type M1 box (Fig. 5d, e and Supplementary Fig. 7c, d). Deletion of the M1 box completely abolished the red light-induced PIF4 expression (Fig. 5f, g). Given the effect of M1 box deletion on pif4 hypocotyl phenotype was less-dramatic than that on PIF4 expression, other signaling pathways may be operated to compensate for the lack of PIF4-mediated hypocotyl elongation in those PPIF4md::PIF4 lines. Delivery of the wild-type pPIF4:: PIF4 cassette to pif4 rescued pif4 hypocotyl thermo-response (Fig. 5h, i and Supplementary Fig. 7e, f). The pPIF4::PIF4 cassette with a deleted M1 box in either pif4 or shb1-D pif4 showed a considerable defect in rescuing pif4 hypocotyl thermo-response but less-dramatic compared with red lightmediated hypocotyl elongation (Fig. 5h, i and Supplementary Fig. 7e, f). The same cassette with a deleted M1 box in either pif4 or shb1-D pif4 also reduced the magnitude of thermoinduced PIF4 expression but did not alter the thermo-induction pattern compared with the wild type cassette (Fig. 5j, k). Therefore, regulated PIF4 expression by SHB1-CCA1/LHY only contributes partially to PIF4-mediated thermo-responses.

Either CCA1 or LHY did not bind in vivo the PIF4 promoter with a deleted M1 box but showed certain affinity toward the base-substituted M1 box (Supplementary Fig. 7g and Supplementary Fig. 8a). In contrast to the in vivo ChIP assays, the $\mathrm{Y} 1 \mathrm{H}$ assays may mask the weak affinity of CCA1 or LHY toward the base-substituted M1 box since $300 \mathrm{ng} / \mathrm{ml} \mathrm{AbA}$ was added in order to titrate off the background growth (Fig. 5b, c). As the basesubstituted M1 box was still partially active in vivo, the disruption of the PIF4-mediated light- and thermo-responses by base substitution was less dramatic compared with M1 box deletion (Supplementary Fig. 7g and Supplementary Fig. 8a-j).
CCA1 and LHY interact with SHB1. SHB1 and CCA1 or LHY were associated with the PIF4 promoter, and CCA1 and LHY were required for the association of SHB1 with the PIF4 promoter. We performed bimolecular fluorescence complementation (BiFC) assays in $N$. benthamiana leaves to test whether SHB1 physically interacts with CCA1 or LHY (Fig. 6a, b). In this system, CCA1 or LHY was fused with YFPN, and SHB1 was fused with $\mathrm{YFPC}^{\mathrm{C}}$. All constructs were driven by the CaMV $35 \mathrm{~S}$ promoter. As controls, CCA1:YFP ${ }^{\mathrm{N}}$ or LHY:YFP ${ }^{\mathrm{N}}$ did not form a fluorescence pair with YFPC, and SHB1:YFPC did not form a fluorescence pair with YFP ${ }^{\mathrm{N}}$ (Fig. 6a). Both CCA1:YFP ${ }^{\mathrm{N}}$ and LHY:YFP ${ }^{\mathrm{N}}$ interacted with SHB1:YFPC as shown in fluorescence images. We also presented confocal images for the interaction between CCA1:YFPN or LHY:YFPN and SHB1:YFPC (Fig. 6b).

We next performed co-immunoprecipitation (co-IP) assays with protein extracts prepared from $35 \mathrm{~S}:: S H B 1: G F P$ and $35 \mathrm{~S}::$ CCA1:FLAG or 35 S::LHY:MYC transgenic Arabidopsis plants (Fig. 6c). SHB1:GFP coprecipitated CCA1:FLAG or LHY:MYC, further confirming their direct physical interaction. The additional bands recognized by anti-GFP antibodies in the SHB1:GFP lane may represent degradation products of full-length SHB1: GFP (Source Data file). We also detected SHB1 interaction with either CCA1 or LHY by BiFC assays in the dark; however, the interaction occurred less frequently (Supplementary Fig. 9a). We performed Co-IP experiments with the 35 S::SHB1:GFP transgenic plants in the dark and under red light, and detected CCA1 or LHY using anti-CCA1 or anti-LHY antibodies (Supplementary Fig. 9b). Equal amounts of CCA1 or LHY were precipitated by SHB1:GFP in the dark and under red light.

SHB1 N terminus interacts with the CCA1 or LHY C terminus. SHB1 contains an N-terminal SPX domain and a C-terminal EXS domain $^{38}$. The SPX domain retains the function of full-length SHB1, whereas the function of the EXS domain remains unknown. SHB1 localizes to the nucleus, and the EXS domain in SHB1 may exhibit a function distinct from those in other SYG1like proteins. To map the interaction domain of SHB1 with CCA1 or LHY, we split SHB1 into the SHB1 N terminus (N520) that contains 520 amino acids and the putative SPX domain and the SHB1 C terminus (C325) that contains 325 amino acids and the EXS domain (Supplementary Fig. 10a). Truncated SHB1 N520 or C325 was fused to YFPC and used in BiFC assays against fulllength CCA1 or LHY that was fused to YFPN ${ }^{\mathrm{N}}$. The N520:YFPC fusion protein formed fluorescence pairs with either CCA1:YFP ${ }^{N}$ or LHY:YFPN in Nicotiana leaves (Supplementary Fig. 10b). The C325: YFPC fusion protein that contains the EXS domain did not interact with CCA1:YFP ${ }^{\mathrm{N}}$ or LHY:YFP ${ }^{\mathrm{N}}$ in the BiFC assays. Both N520:YFPC and C325: YFPC fusion proteins were probed with anti-HA antibodies and expressed at comparable levels (Supplementary Fig. 12a).

CCA1 and LHY are MYB transcription factors and contain a conserved MYB DNA-binding domain at their $\mathrm{N}$ termini that is approximately located from amino acids 22-72. We made constructs in which the N-terminal 173 amino acids of CCA1 or LHY were fused to YFP ${ }^{\mathrm{N}}\left(\mathrm{N} 173: \mathrm{YFP}^{\mathrm{N}}\right)$ or the C-terminal sequence after amino acid 173 was fused to YFPN (CCA1 C435: YFP $^{\mathrm{N}}$ or LHY C487:YFP ${ }^{\mathrm{N}}$ ) (Supplementary Fig. 11a). The C terminus but not the N-terminal MYB binding domains of CCA1 or LHY interacted with full-length SHB1 in the BiFC assays (Supplementary Fig. 11b). Various controls were also included to support the specificity of the interactions. Of the total proteins probed with anti-Myc antibodies, CCA1 or LHY N173:YFPN, CCA1 C435:YFPN and LHY C487:YFP ${ }^{\mathrm{N}}$ fusion proteins were expressed at comparable levels (Supplementary Fig. 12b). 
a

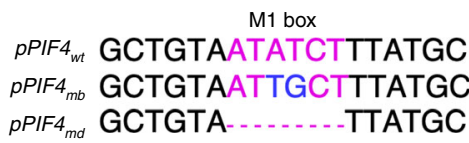

b

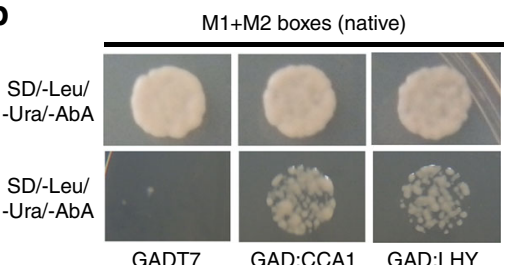

C
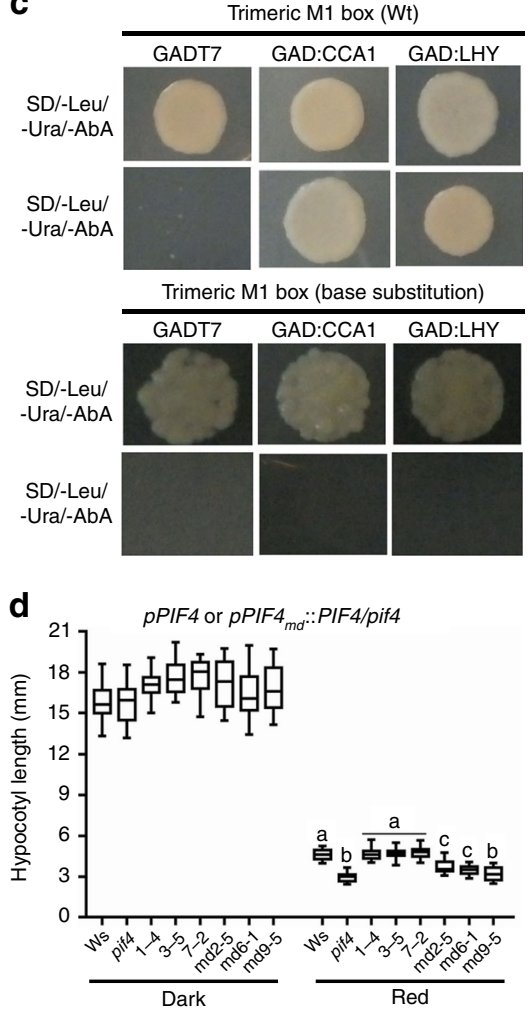
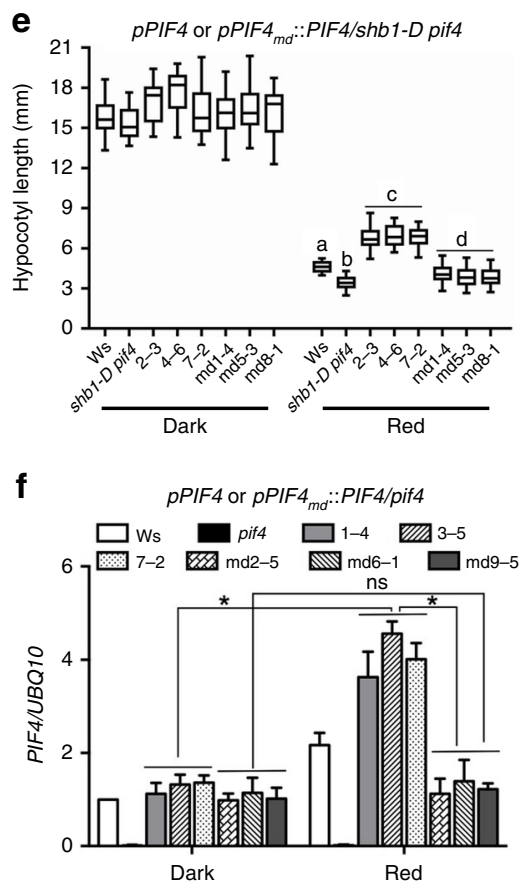

g

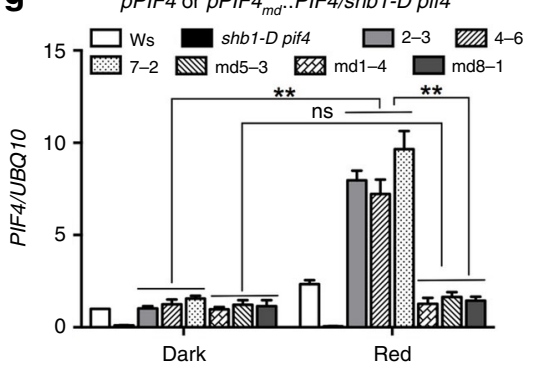

h
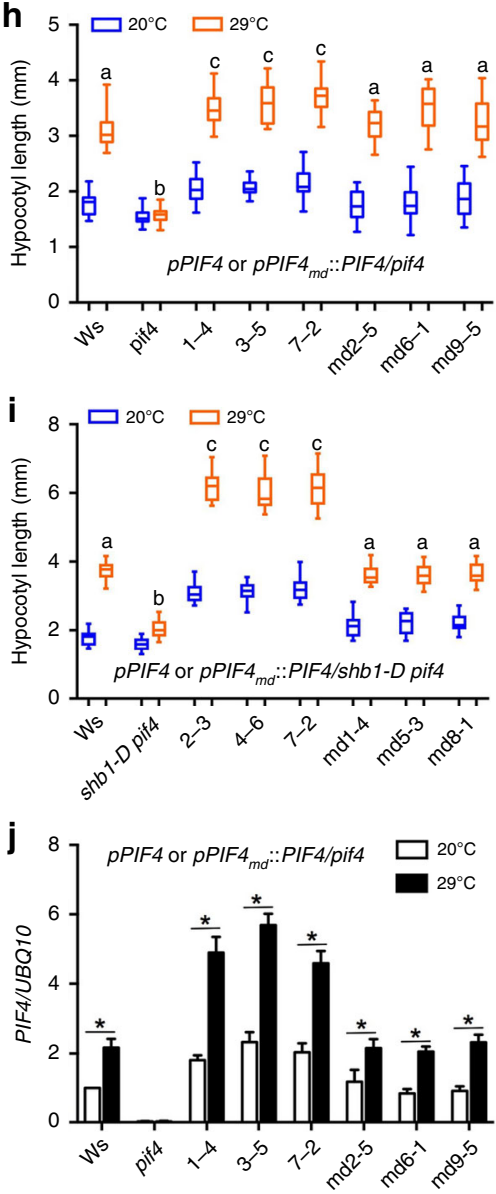

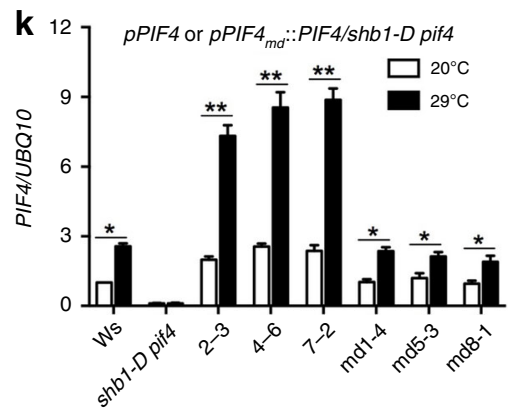

Fig. 5 CCA1 and LHY recognize MYB M1 box in the PIF4 promoter. a Potential MYB-binding element M1 is noted in red, and the bases mutated are noted in blue. $\mathrm{mb}$ and $\mathrm{md}$ indicate mutation by base substitution and deletion, respectively. Yeast one-hybrid assays for CCA1 or LHY in pGADT7 over a 424 -bp fragment that contains both M1 and M2 elements from the PIF4 promoter in the pAbAi vector $\mathbf{b}$ and for CCA1 or LHY in pGADT7 over trimeric repeats of wild type (upper) or mutated (lower) M1 element c. Golden Y1H cells were grown on SD/-Leu/-Ura media with or without $300 \mathrm{ng} / \mathrm{ml}$ Aureobasidin A (AbA) selection. Hypocotyl lengths of Ws, pif4 and pPIF4 or pPIF4md::PIF4 in pif4 $\mathbf{d}$ and Ws, shb1-D pif4 and pPIF4 or pPIF4md::PIF4 in shb1-D pif4 e. PIF4 expression in Ws, pif4 and pPIF4 or pPIF4md::PIF4 in pif4 $\mathbf{f}$ and Ws, shb1-D pif4 and pPIF4 or pPIF4md::PIF4 in shb1-D pif4 $\mathbf{g}$. The seedlings were grown in the dark or under $10 \mu \mathrm{mol} \mathrm{m} \mathrm{m}^{-2} \mathrm{~s}^{-1}$ red light for 4 days. Hypocotyl lengths of Ws, pif4 and pPIF4 or pPIF4md::PIF4 in pif4 $\mathbf{h}$ and Ws, shb1-D pif4 and pPIF4 or pPIF4md::PIF4 in shb1-D pif4 i under $30 \mu \mathrm{mol} \mathrm{m} \mathrm{m}^{-2} \mathrm{~s}^{-1}$ white light at $20^{\circ} \mathrm{C}$ for 7 days or $20^{\circ} \mathrm{C}$ for 4 days followed by $29^{\circ} \mathrm{C}$ for 3 days. PIF4 expression in Ws, pif4 and pPIF4 or pPIF4md::PIF4 in pif4 $\mathbf{j}$ and Ws, shb1-D pif4 and pPIF4 or pPIF4md::PIF4 in shb1-D pif4 $\mathbf{k}$ grown at $20^{\circ} \mathrm{C}$ for 5 days and then incubated at $20^{\circ} \mathrm{C}$ or $29^{\circ} \mathrm{C}$ for $4 \mathrm{~h}$. Significance levels by Student's two-tailed heteroscedastic $t$ tests in $\mathbf{d}: p<0.001$ between $\mathbf{a}$ and $\mathbf{b}, p<0.01$ between $\mathbf{a}$ and $\mathbf{c}, p<0.05$ between $\mathbf{b}$ and $\mathbf{c}$; in e: $p<0.001$ between $\mathbf{a}$ and $\mathbf{b}$, $\mathbf{a}$ and $\mathbf{c}, \mathbf{b}$ and $\mathbf{c}$ or $\mathbf{c}$ and $\mathbf{d}, p<0.01$ between $\mathbf{a}$ and $\mathbf{d}, p<0.05$ between $\mathbf{b}$ and $\mathbf{d} ;$ in $\mathbf{h}: p<0.001$ between $\mathbf{a}$ and $\mathbf{b}$ or $\mathbf{b}$ and $\mathbf{c}, p<0.01$ between $\mathbf{a}$ and $\mathbf{c}$; in $\mathbf{i}: p<0.001$ between $\mathbf{a}$ and $\mathbf{b}$, $\mathbf{a}$ and $\mathbf{c}$ or $\mathbf{b}$ and $\mathbf{c}$. Source data are provided as a Source Data file

CCA1 and LHY co-act with SHB1. SHB1 and CCA1 or LHY were required for PIF4 expression giving that knocking down of SHB1 or knocking out of CCA1/LHY reduced PIF4 expression (Fig. 1a, e). We conducted in vivo trans-activation experiments in Arabidopsis ccal thy protoplasts with various constructs (Fig. 6d). We used a PIF4 promoter::LUC reporter along with a CaMV 35 S::REN residing on the same vector to control differences in transformation efficiency. We delivered CCA1 and LHY driven by their native promoter and SHB1 driven by $35 \mathrm{~S}$ promoter as effectors to ccal lhy (Fig. 6d). We used the 35 $\mathrm{S}$ promoter for SHB1 giving that its native promoter drives a very low level of expression and SHB1 is not rhythmically expressed (Supplementary Fig. 1c). Compared with controls without any effector proteins delivered, delivery of the $p C C A 1:$ : 
a

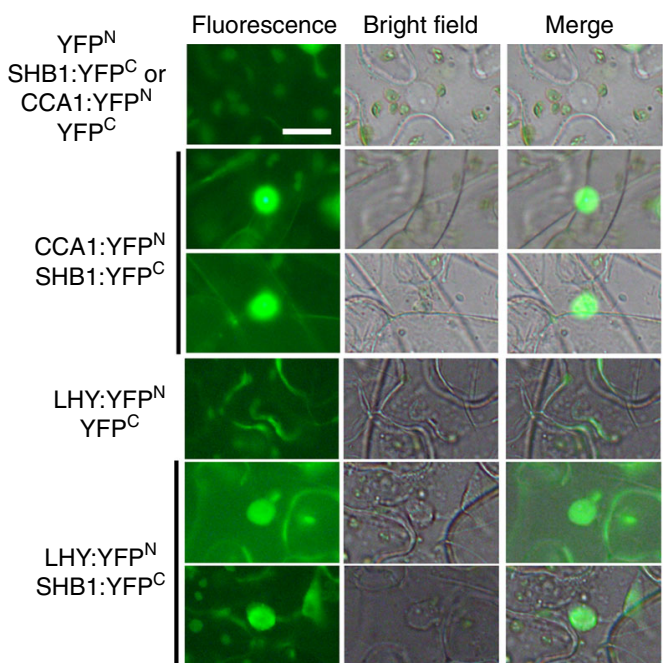

b
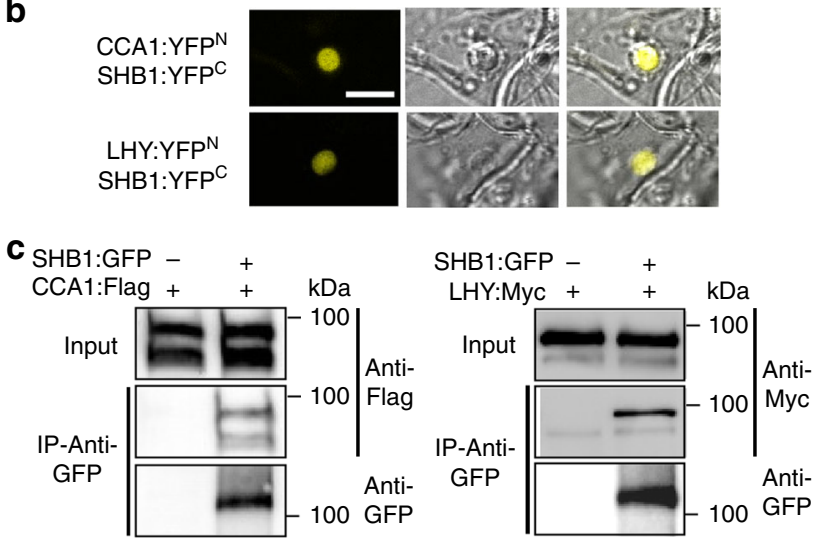

d

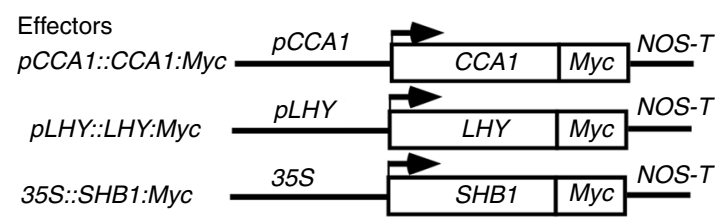

Reporter

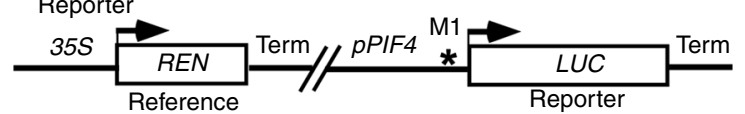

e
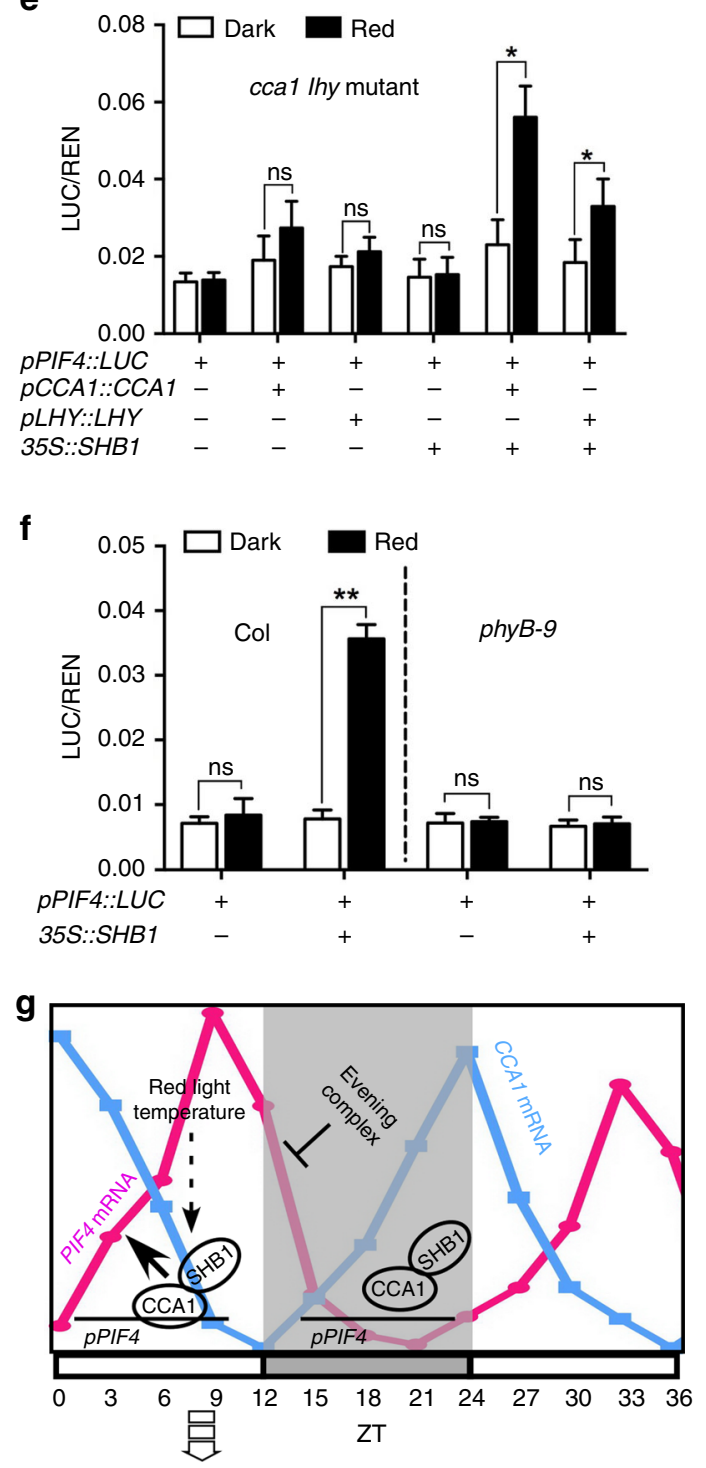

Optimum photomorphogenesis

Enhanced thermomorphogenesis

Fig. 6 SHB1 co-acts with CCA1 and LHY. a BiFC fluorescent images of YFPN with SHB1:YFPC, CCA1:YFPN or LHY:YFPN with YFPC, and CCA1:YFPN or LHY: YFPN with SHB1:YFPC. b BiFC confocal images of CCA1:YFPN or LHY:YFPN with SHB1:YFPC. Images were captured 48-72 h in Nicotiana leaf epidermal cells after Agrobacteria transfection. Bar $=10 \mu \mathrm{m}$. c Co-immunoprecipitation (Co-IP) of CCA1:FLAG or LHY:MYC by SHB1:GFP in protein extracts prepared from transgenic Arabidopsis with anti-GFP antibody. d Effector, reporter, and reference constructs used. Arrowheads indicate transcription start sites, and NOS-T represents polyadenylation signal from the nopaline synthase gene. Asterisk indicates the location of the M1 element in the PIF4 promoter. Relative LUC/ REN activity in cca1 Ihy transformed with a reporter along with pCCA1::CCA1:MYC, pLHY::LHY:MYC or 35 S::SHB1:MYC e and in Col or phyB-9 transformed with reporter in the absence or presence of 35 S::SHB1:MYC $\mathbf{f}$ in the dark or under red light. Source data are provided as a Source Data file. $\mathbf{g}$ A model explaining the regulation of CCA1 or LHY and SHB1 over PIF4 expression. PIF4 expression is repressed by the evening complex (EC) or PRRs at early evening. Toward the end of night and after dawn, CCA1 recruits SHB1 to mediate red light induction of PIF4 expression. This constitutes a signaling loop to optimize photomorphogenesis and enhance thermomorphogenesis

CCA1:MYC or pLHY::LHY:MYC construct alone to ccal lhy barely activated the expression of $L U C$ from the PIF4 promoter either in the dark or under red light (Fig. 6e). Delivery of the 35 $S:: S H B 1: M Y C$ construct alone to ccal hy also exhibited minimal effects on the transcription of LUC from the PIF4 promoter.

When $p C C A 1:: C C A 1: M Y C$ and 35 S::SHB1:MYC were codelivered to ccal lhy, the transcription of LUC from the PIF4 promoter was activated weakly in the dark but strongly under red light (Fig. 6e). The effects on $L U C$ transcription from the
PIF4 promoter were considerably less dramatic when 35 S:: SHB1:MYC was codelivered with $p L H Y:: L H Y: M Y C$ either in the dark or under red light. CCA1 protein levels were comparable to that of LHY protein (Supplementary Fig. 12c). CCA1 or LHY likely recruits SHB1 to the PIF4 promoter, and SHB1 mediates red light-induced PIF4 expression. This red light induction was blocked in phyB-9 under red light (Fig. 6f). The levels of SHB1, CCA1, and LHY proteins were not different between transformed protoplasts maintained in the dark or under red light (Supplementary Fig. 12c). The nuclear localization of SHB1: 
GFP was not affected in the dark compared with that under red light (Supplementary Fig. 12d).

\section{Discussion}

Light and temperature are two important environmental signals that regulate plant growth and development. However, excess light often damages the photosynthetic apparatus and frequently causes photoinhibition. Plants thus evolve a series of strategies to achieve optimum photomorphogenesis. Here we demonstrate a regulatory desensitization step involving SHB1, CCA1/LHY, and PIF4. We proposed a model explaining how CCA1/LHY and SHB1 upregulate PIF4 expression (Fig. 6g). Our study suggests that the highly expressed circadian clock proteins CCA1 and LHY in the morning recruits SHB1 to activate red light-induced PIF4 expression and desensitize light responses. PIF4 is also a positive regulator of thermomorphogenesis. When temperature increases after morning, the interaction of SHB1 with CCA1 may sustain PIF4 expression to allow plants to better adapt to the temperature-increasing environment. Alternatively, PIF4 transcription allows plants to monitor day-time shade and temperature, which eventually influences PIF4 protein accumulation and activity.

The EC formed by LUX, ELF3, and ELF4 proteins peaks at dusk, binds to the PIF4 promoter via the LUX transcription factor, and represses PIF4 transcription in the early evening ${ }^{46}$. EC may recognize two binding elements near the CCA1 and LHY-binding elements. The occupation of the elements by EC and possibly PRRs in the dark may interfere with the binding of CCA1 and LHY to the PIF4 promoter. Indeed, CCA1 and LHY were not associated with the PIF4 promoter in the dark (Fig. 4c and Supplementary Fig. 4c). Near dawn, CCA1 and LHY associate with the PIF4 promoter, recruit SHB1 to activate PIF4 transcription and promote growth, such as hypocotyl elongation (Fig. $6 \mathrm{~g}$ ). CCA1 was originally discovered as an activator of a dawn gene $\mathrm{LHCB}^{12}$. CCA1 and LHY also repress the evening element-containing genes at dawn ${ }^{49}$. We discovered a new role of CCA1 and LHY to directly recruit SHB1 that mediates red light-induced expression of PIF4 in the morning and thereafter. As such, PIF4 transcription peaks after the middle of the day.

ChIP-seq analysis identified the regions of CCA1 occupancy at many EE-containing, evening-expressed, clock-regulated genes in the Arabidopsis genome ${ }^{48}$. CCA1 recognition elements are also located near many genes with peak expression in the morning and in proximity to genes that do not cycle in LL conditions. CCA1 recognizes the canonical EE (AAATATCT) in LL conditions, and the binding capacity of CCA1 is more affected by alteration in the TCT sequence compared with alterations in the ATA or $\mathrm{AA}^{50}$. In our yeast one-hybrid analysis, CCA1 only recognizes the M1 element sequence ATATCT, not the M2 element sequence ATATCA in the PIF4 promoter (Fig. 5a, $\mathrm{c}$ and Supplementary Fig. 6). The last base in the core sequence is apparently critical for CCA1 recognition. In addition, the flanking sequences may also affect the affinity of CCA1 to its binding elements.

PIFs belong to a large family of basic helix-loop-helix (bHLH) proteins and exhibit a variety of different functions ${ }^{51}$. Monogenic pif3, pif4, pif5, and pif7 null mutants exhibit light-hypersensitive seedling phenotypes, whereas PIF4, PIF5, and PIF7 are clock regulated $^{8,52}$. The three PIFs promote the elongation of hypocotyls and petioles during vegetative growth in a redundant manner ${ }^{39,53-55}$. PIF4 has a more-prominent role than PIF5 ${ }^{56,57}$. Among the PIF genes, SHB1 specifically regulates the red lightinduced expression of PIF4 and likely PIF7 (Fig. 1a and Supplementary Fig. 1a). Light-activated phyB interacts with PIF4 and targets it for post-transcriptional degradation following the entire light period ${ }^{53,58,59}$. In these experiments, a CaMV $35 \mathrm{~S}$ promoterdriven PIF4 construct was used. On the other hand, PIF4 reaccumulates after a few hours of light treatment ${ }^{60,61}$. When PIF4 was driven by its native promoter in a recent study, PIF4 protein accumulated in the light period from ZT0 to ZT8 but not in the dark period from ZT12 to ZT20 ${ }^{42}$. Despite continuous PIF4 degradation, the upregulation of PIF4 expression by CCA1 and SHB1 throughout the day might be responsible for the reappearance of PIF4 protein, constituting a negative regulatory step to antagonize photomorphogenesis.

\section{Methods}

Plant materials and growth conditions. Arabidopsis thaliana ecotype Columbia-0 (Col) and Wassilewskija (Ws) were used as wild-type plants. The mutant lines shb1, shb1-D, and pif4 were described previously ${ }^{37,53}$. cca1-11, cca1-11 lhy-21, and ELF4:GFP seeds were from Gang Li, and $l h y-21$ seeds were from ABRC (https:// abrc.osu.edu/). The shb1-D pif4 double mutant was generated by crossing shb1-D to pif4 and PCR-genotyped using the primers described previously ${ }^{37}$. shbl ccal lhy or shb1-D ccal lhy triple mutant was generated by crossing $\operatorname{shbl}$ or shb1-D to ccal lhy double mutant and PCR-genotyped using the primers listed in table S1. All plants were grown in a growth room with a $16 \mathrm{~L} / 8 \mathrm{D}$ cycle at $22^{\circ} \mathrm{C}$ for seed propagation. For circadian expression analysis, Arabidopsis seedlings were entrained under a 12-hr white or red light and 12-hr dark cycle for 7 days, and then released to continuous white or red light for the following days as described in the figure legends. Similar results were observed when seedlings were released to continuous white or red light for one day and then sampled for the following days.

Quantitative RT-PCR. For quantitative reverse transcription-polymerase chain reaction (RT-PCR) analysis, total RNA was extracted from seedlings using the MiniBEST Plant RNA Extraction Kit (Takara) or the SV Total RNA Isolation Kit (Promega). SuperScript ${ }^{\text {th }}$ II Reverse Transcriptase (Invitrogen) was used to synthesize cDNA from the RNA. Quantitative real-time PCR was performed with the TransStart Tip Green qPCR SuperMix (Transgen Biotech) on a QuantStudio ${ }^{\text {TM }} 6$ Flex Real-Time PCR machine. The thermal cycling program was $95^{\circ} \mathrm{C}$ for $5 \mathrm{~min}$, followed by 40 cycles of $95^{\circ} \mathrm{C}$ for $10 \mathrm{~s}, 56^{\circ} \mathrm{C}$ for $10 \mathrm{~s}$ and $72^{\circ} \mathrm{C}$ for $20 \mathrm{~s}$. The last step involves a one-cycle dissociation stage at $95^{\circ} \mathrm{C}$ for $5 \mathrm{~s}, 65^{\circ} \mathrm{C}$ for $1 \mathrm{~min}, 98^{\circ} \mathrm{C}$ for $1 \mathrm{~min}$ and $40^{\circ} \mathrm{C}$ for $10 \mathrm{~s}$. Most RT-PCR were performed with three biological replicates unless it is specifically indicated in the figure legends. Each biological replicate was represented by three technical replicates. The expression levels of specific genes were normalized to that of $U B Q 10$ and were presented relative to the expression levels in wild type. The gene-specific primers for qRT-PCR analysis are listed in Supplementary Table 1.

Hypocotyl length measurement. Seeds were sterilized by $1 \%(\mathrm{v} / \mathrm{v})$ sodium hypochloride and $0.2 \%(\mathrm{v} / \mathrm{v})$ sodium dodecyl sulphate (SDS) and plated on $1 / 2 \mathrm{MS}$ medium. After a 3-day vernalization at $4{ }^{\circ} \mathrm{C}$, seeds were treated with white light for $2-3 \mathrm{~h}$ and then incubated under $10 \mu \mathrm{mol} \mathrm{m}^{-2} \mathrm{~s}^{-1}$ red light for 4 days. For thermomorphogenesis, Arabidopsis seedlings were grown under continuous fluorescent white light $\left(30 \mu \mathrm{mol} \mathrm{m}^{-2} \mathrm{~s}^{-1}\right)$ at $20^{\circ} \mathrm{C}$ for 7 days or at $20^{\circ} \mathrm{C}$ for 4 days followed by growth at $29^{\circ} \mathrm{C}$ for 3 days. More than 50 seedlings were photoimaged and hypocotyl lengths were measured using ImageJ software (http://rsb.info.nih.gov/ij). Hypocotyl experiments were repeated thrice, and one representative dataset was shown. About 50 seedlings were measured for each genotype. For PIF4 expression analysis at $29^{\circ} \mathrm{C}$, seedlings were grown at $20^{\circ} \mathrm{C}$ for 5 days and then incubated at either $20^{\circ} \mathrm{C}$ or $29^{\circ} \mathrm{C}$ for $4 \mathrm{hr}$ before total RNA was extracted.

Plasmid construction. All primers used to make plasmid constructs were listed in Supplementary Table 1. All constructs, except for the plasmids used for yeast onehybrid $(\mathrm{Y} 1 \mathrm{H})$ assay, were made by using the gateway system. In general, the fulllength genomic coding region was PCR amplified from Col genomic DNA, cloned into the $\mathrm{pCR} 8 / \mathrm{GW} / \mathrm{TOPO} \mathrm{TA}$ vector, and then recombined into the corresponding destination vectors. To examine the in vivo function of M1 element in PIF4 promoter, a genomic fragment that contains PIF4 promoter up to -1524 bp and coding sequence was cloned into the TA vector. This TA clone was then used as template to mutate the M1 element as base substitution or deletion by a reversePCR procedure (TOYOBO, SMK-101). Transgenes were verified by using PIF4-F and $\mathrm{c}-\mathrm{Myc}-\mathrm{R}$ primers.

Protein isolation and western blot. To detect rhythmic PIF4 accumulation in Col, shb1, Ws, and shb1-D, seedlings were first grown under $12-\mathrm{hr} 2 \mu \mathrm{mol} \mathrm{m}{ }^{-2} \mathrm{~s}^{-1}$ red light and 12-hr dark for 5 days and then sampled at ZT0 and every $3 \mathrm{~h}$ thereafter for $24 \mathrm{~h}$. Protein isolation buffer contains $100 \mathrm{~mm}$ MOPS pH 7.6, $100 \mathrm{~mm} \mathrm{NaCl}$, $10 \%$ glycerol, $40 \mathrm{~mm} 2$-mercaptoethanol, $5 \%$ SDS, $1 \times$ protease inhibitor cocktail from Roche, and $2 \mathrm{~mm}$ phenylmethylsulfonyl fluoride (PMSF). Eighty $\mu \mathrm{l}$ buffer were added to $100 \mu \mathrm{g}$ grinded powder. The mixture was immediately heated at $70{ }^{\circ} \mathrm{C}$ for $10 \mathrm{~min}$ and separated on $8 \%$ SDS-PAGE gel. PIF4 protein was monitored 
by western blot using anti-PIF4 antibody AS16 3955 (Agrisera, Sweden) from three biological replicates. Protein quantification was performed with IMAGEJ normalized to an actin band.

To examine the accumulation of tagged proteins in transgenic plants, the proteins were isolated in buffer that contained $50 \mathrm{~mm}$ Tris- $\mathrm{HCl} \mathrm{pH} 8.0,10 \mathrm{~mm}$ EDTA, $1 \%$ SDS, $1 \mathrm{~mm}$ PMSF and $1 \times$ protease inhibitors (Roche). The tagged proteins were detected in western blot using anti-GFP ab1218 (Abcam, Cambridge, MA), anti-FLAG ab49763 (Abcam, Cambridge, MA) and anti-MYC ab32 (Abcam, Cambridge, MA) antibodies. To examine the accumulation of tagged proteins in transient assays, the proteins were isolated in buffer that contained $50 \mathrm{~mm}$ Tris- $\mathrm{HCl}$ $\mathrm{pH} 7.5,150 \mathrm{~mm} \mathrm{NaCl}, 0.1 \% \mathrm{NP}-40,4 \mathrm{~m}$ urea, and $1 \mathrm{~mm}$ PMSF. The tagged proteins were detected by anti-HA CW0092 (CWBIO, China) and anti-MYC antibodies as described above. Total proteins in Supplementary Fig. 12c were extracted from protoplasts by boiling in $2 \times$ SDS loading buffer at $95^{\circ} \mathrm{C}$ for $10 \mathrm{~min}$. The tagged proteins were detected using anti-GFP and anti-MYC antibodies as described above.

ChIP assay. ChIP assays were performed as described previously ${ }^{44,62}$. Seedlings that have 35 S::SHB1:GFP, 35 S::CCA1:FLAG or 35 S::LHY:MYC transgene were grown in the dark or under $15 \mu \mathrm{mol} \mathrm{m}^{-2} \mathrm{~s}^{-1}$ red light for 5 days, and then crosslinked for $30 \mathrm{~min}$ in $1 \%$ formaldehyde solution under vacuum. For rhythmic ChIP analysis, Col wild type and 35 S::SHB1:GFP transgenic plants were grown under 12-hr red light and 12-hr dark for 3-weeks, and sampled at ZT0 and every $3 \mathrm{~h}$ thereafter for $24 \mathrm{~h}$. The plant materials were ground for $10 \mathrm{~min}$ on ice in nuclear isolation buffer with $1 \%$ formaldehyde, and cross-linking was quenched by adding glycine to a final concentration of $125 \mathrm{~mm}$. The chromatin complex was isolated by nuclear lysis buffer (50 mM Tris- $\mathrm{HCl}$ pH 8.0, $10 \mathrm{~mm}$ EDTA, 0.5\% SDS, $0.1 \mathrm{~mm}$ PMSF and $1 \times$ protease inhibitors) and sheared by sonication to generate fragments that were $\sim 300-500 \mathrm{bp}$. The sonicated chromatin complex was then immunoprecipitated by anti-GFP, anti-FLAG, and anti-MYC antibodies as previously described. For rhythmic ChIP analysis, the sonicated chromatin complex was harvested by anti-CCA1 R1234-3 (Abiocode, Agoura Hills, CA), anti-LHY R3095-2 (Abiocode, Agoura Hills, CA) and anti-GFP antibodies. After the reversal of cross-linking, DNA was precipitated in the presence of glycogen (Thermo Fisher) and analyzed by ChIP-qPCR. Most ChIP-PCR were performed with three biological replicates unless it is specifically indicated in the figure legends. Each biological replicate was represented by three technical replicates. The fold enrichment of the specific chromatin fragment was normalized to the UBQ10 amplicon. All primers used for ChIP-qPCR were listed in Supplementary Table 1.

Yeast one-hybrid (Y1H) assay. A native 424-bp DNA fragment in the PIF4 promoter was PCR amplified and cloned into the Hind III and Sal I sites of the pAbAi vector (Clontech). Three tandem copies of the putative CCA1/LHY-binding element M1 or M2 were synthesized as oligonucleotides and ligated into the Hind III and Sal I sites of the $p A b A i$ vector (Clontech). Each repeat of the element has a GCTGTAATATCTTTATGC or TTCCACATATCAGGTTAT sequence with a 6bp flanking sequence on either side of the core elements. The $p A b A i$ vectors harboring the constructs were integrated into the genome of the $\mathrm{Y} 1 \mathrm{H}$ Gold yeast strain. The coding sequences of CCA1 and $L H Y$ were PCR amplified from CDNA generated from Arabidopsis Col total RNA and cloned into the pGADT7-AD vector (Yeast Protocols Handbook by Clontech, Mountain View, CA). Transformants were selected on minimal synthetic dropout (SD) medium lacking Leu, Trp, and Ura. Yeast cells grown in SD/-Leu/-Trp/-Ura broth were diluted to $\mathrm{OD}_{600} 0.5$ and plated on $\mathrm{SD} /$-Leu/-Trp/-Ura plate with or without $\mathrm{AbA}$ as described by the Matchmaker Gold Yeast One-Hybrid Library Screening System Protocol (Clontech, Mountain View, CA).

BiFC assay. BiFC experiments were conducted as described ${ }^{63}$. To generate the BiFC constructs, CCA1 or LHY cDNA and SHB1 genomic sequence without their stop codons were PCR amplified and subcloned into the binary vectors $p S P Y N E$ and $P S P Y C E$ under the control of the cauliflower mosaic virus (CaMV) $35 \mathrm{~S}$ promoter. pSPYNE::CCA1 or LHY was co-transformed with pSPYCE::SHB1 into $N$. benthamiana leaves by agroinfiltration. Their interactions in $N$. benthamiana leaves were analyzed using an Olympus fluorescence microscope (Olympus BX53 with a DP26 CCD camera) or a confocal microscope (Leica TCS SP5 II).

Co-IP assay. For co-IP assays, total protein of 35 S::SHB1:GFP and 35 S::CCA1: FALG or 35 S::LHY:MYC seedlings was extracted using IP buffer (10\% Glycerol, 25 mм Tris- $\mathrm{HCl}$ pH 7.5, 1 mм EDTA, $150 \mathrm{~mm} \mathrm{NaCl}, 0.1 \%$ Triton-100, 2 mм $\beta$ mercaptoethanol, $1 \mathrm{~mm}$ PMSF and $1 \times$ protease inhibitors). After centrifugation at $13,000 \mathrm{rpm}$ for $20 \mathrm{~min}$, the two sources of proteins were mixed and incubated for $2-3 \mathrm{~h}$ at $4{ }^{\circ} \mathrm{C}$. The mixture was then incubated with the anti-GFP rabbit polyclonal antibody 10004D (Thermo Fisher, CA) for another $2 \mathrm{~h}$. The beads were washed four times with IP buffer. The pelleted beads were boiled in $60 \mu \mathrm{l} 2 \times$ SDS buffer and separated by $8 \%$ SDS-PAGE. Western blots were probed by using anti-FLAG or anti-MYC antibodies at a 1:1000 dilution.

Transient trans-activation assay. Arabidopsis mesophyll protoplast isolation and transformation were performed as described ${ }^{64}$. pCCA1::CCA1:MYC, pLHY::LHY:
MYC and 35 S::SHB1:MYC were used in effector constructs. The $p$ Green $I I-0800-$ LUC vector that bears PPIF4::LUC and 35 S::REN was used as a reporter construct. Luciferase activity was measured using the Dual-Luciferase Reporter Assay System (Promega, USA). Relative luciferase activity was normalized to REN activity as a LUC/REN ratio for each biological sample. Experiments were repeated thrice and each biological replicate was represented by three technical replicates.

\section{Data availability}

The authors declare that all data supporting the findings of this study are available within the paper and its supplementary information files. The source data underlying Figs. 1, 2, 3a-d, f, 4b-e, 5d-k, and 6c, e, f and Supplementary Figs. 1a-c, 2a-c, g, 4a-f, 5a, b, 7a, b, g, $8 \mathrm{~b}-\mathrm{j}, 9 \mathrm{~b}$, and $12 \mathrm{a}-\mathrm{c}$ are provided as a Source Data file.

Received: 8 December 2017 Accepted: 18 June 2019

Published online: 15 July 2019

\section{References}

1. Schäfer, E. \& Nagy, F. Function and signal transduction mechanisms. Photomorphogenesis in Plants and Bacteria. 3rd edn (2006).

2. Sharrock, R. A. \& Quail, P. H. Novel phytochrome sequences in Arabidopsis thaliana: structure, evolution, and differential expression of a plant regulatory photoreceptor family. Genes Dev. 3, 1745-1757 (1989)

3. Clack, T., Mathews, S. \& Sharrock, R. A. The phytochrome apoprotein family in Arabidopsis is encoded by five genes: the sequences and expression of PHYD and PHYE. Plant Mol. Biol. 25, 413-427 (1994).

4. Franklin, K. A. \& Quail, P. H. Phytochrome functions in Arabidopsis development. J. Exp. Bot. 61, 11-24 (2010).

5. Bauer, D. et al. Constitutive photomorphogenesis 1 and multiple photoreceptors control degradation of phytochrome interacting factor 3, a transcription factor required for light signaling in Arabidopsis. Plant Cell 16, 1433-1445 (2004).

6. Park, E. et al. Degradation of phytochrome interacting factor 3 in phytochrome-mediated light signaling. Plant Cell Physiol. 45, 968-975 (2004).

7. Shen, H., Moon, J. \& Huq, E. PIF1 is regulated by light-mediated degradation through the ubiquitin/26S proteasome pathway to optimize seedling photomorphogenesis in Arabidopsis. Plant J. 44, 1023-1035 (2005).

8. Al-Sady, B., Ni, W., Kircher, S., Schäfer, E. \& Quail, P. H. Photo-activated phytochrome induces rapid PIF3 phosphorylation prior to proteasomemediated degradation. Mol. Cell 23, 439-446 (2006)

9. Leivar, P. \& Quail, P. H. PIFs: pivotal components in a cellular signaling hub. Trends Plant Sci. 16, 19-28 (2011).

10. Leivar, P. \& Monte, E. PIFs: systems integrators in plant development. Plant Cell 26, 56-78 (2014).

11. Castillon, A., Shen, H. \& Huq, E. Phytochrome interacting factors: central players in phytochrome-mediated light signaling networks. Trends Plant Sci. 12, 514-521 (2007)

12. Wang, Z. Y. \& Tobin, E. M. Constitutive expression of the CIRCADIAN CLOCK ASSOCIATED 1 (CCA1) gene disrupts circadian rhythms and suppresses its own expression. Cell 93, 1207-1217 (1998).

13. Schaffer, R. et al. The late elongated hypocotyl mutation of Arabidopsis disrupts circadian rhythms and the photoperiodic control of flowering. Cell 93, 1219-1229 (1998).

14. Alabadí, D. et al. Reciprocal regulation between TOC1 and LHY/CCA1 within the Arabidopsis circadian clock. Science 293, 880-883 (2001).

15. Alabadí, D., Yanovsky, M. J., Más, P., Harmer, S. L. \& Kay, S. A. Critical role for CCA1 and LHY in maintaining circadian rhythmicity in Arabidopsis. Curr. Biol. 12, 757-761 (2002).

16. Gendron, J. M. et al. Arabidopsis circadian clock protein, TOC1, is a DNA binding transcription factor. Proc. Natl Acad. Sci. USA. 109, 3167-3172 (2012).

17. Huang, W. et al. Mapping the core of the Arabidopsis circadian clock defines the network structure of the oscillator. Science 336, 75-79 (2012).

18. Pokhilko, A. et al. The clock gene circuit in Arabidopsis includes a repressilator with additional feedback loops. Mol. Syst. Biol. 8, 574-587 (2012).

19. Covington, M. F. \& Harmer, S. L. The circadian clock regulates auxin signaling and responses in Arabidopsis. PLoS Biol. 5, e222 (2007).

20. Rawat, R. et al. REVEILLE8 and PSEUDO-REPONSE REGULATOR5 form a negative feedback loop within the Arabidopsis circadian clock. PLoS Genet. 7, e1001350 (2011).

21. Wu, J. F., Wang, Y. \& Wu, S. H. Two new clock proteins, LWD1 and LWD2, regulate Arabidopsis photoperiodic flowering. Plant Physiol. 148, 948-959 (2008).

22. Wang, Y. et al. LIGHT-REGULATEDWD1 and PSEUDO-RESPONSE REGULATOR9 form a positive feedback regulatory loop in the Arabidopsis circadian clock. Plant Cell 23, 486-498 (2011). 
23. Maria, A. K. et al. High temperature-mediated Adaptations in plant architecture require the bHLH transcription factor PIF4. Curr. Biol. 19 408-413 (2009).

24. Zhu, J. Y., Oh, E., Wang, T. \& Wang, Z. Y. TOC1-PIF4 interaction mediates the circadian gating of thermoresponsive growth in Arabidopsis. Nat. Commun. 7, 13692 (2016).

25. Quint, M. et al. Molecular and genetic control of plant thermomorphogenesis. Nat. Plants 6, 15190 (2016).

26. Gray, W. M., Ostin, A., Sandberg, G., Romano, C. P. \& Estelle, M. High temperature promotes auxin-mediated hypocotyl elongation in Arabidopsis. Proc. Natl Acad. Sci. USA. 95, 7197-7202 (1998).

27. Oh, E., Zhu, J. Y. \& Wang, Z. Y. Interaction between BZR1 and PIF4 integrates brassinosteroid and environmental responses. Nat. Cell Biol. 14, 802-809 (2012).

28. Oh, E. et al. Cell elongation is regulated through a central circuit of interacting transcription factors in the Arabidopsis hypocotyl. Elife 3, e03031 (2014).

29. Sun, J., Qi, L., Li, Y., Chu, J. \& Li, C. PIF4-mediated activation of YUCCA8 expression integrates temperature into the auxin pathway in regulating Arabidopsis hypocotyl growth. PLoS Genet. 8, e1002594 (2012).

30. Franklin, K. A. et al. Phytochrome-interacting factor 4 (PIF4) regulates auxin biosynthesis at high temperature. Proc. Natl Acad. Sci. USA. 108, 20231-20235 (2011).

31. Aro, E. M., Virgin, I. \& Andersson, B. Photoinhibition of Photosystem II. Inactivation, protein damage and turnover. Biochim. Biophys. Acta 1143, 113-134 (1993).

32. Powles, S. B. Photoinhibition of photosynthesis induced by visible light. Annu. Rev. Plant Physiol. 35, 15-44 (1984).

33. Shulaev, V. \& Oliver, D. J. Metabolic and proteomic markers for oxidative stress. New tools for reactive oxygen species research. Plant Physiol. 141, 367-372 (2006).

34. Zeng, X. Q., Chow, W. S., Su, L. J., Peng, X. X. \& Peng, C. L. Protective effect of supplemental anthocyanins on Arabidopsis leaves under high light. Physiol. Plant 138, 215-225 (2010).

35. Gururani, M. A., Venkatesh, J. \& Tran, L. S. Regulation of photosynthesis during abiotic stress-induced photoinhibition. Mol. Plant 8, 1304-1320 (2015).

36. Erickson, E., Wakao, S. \& Niyogi, K. K. Light stress and photoprotection in Chlamydomonas reinhardtii. Plant J. 82, 449-465 (2015).

37. Kang, X. \& Ni, M. Arabidopsis SHORT HYPOCOTYL UNDER BLUE1 contains SPX and EXS domains and acts in cryptochrome signaling. Plant Cell 4, 921-934 (2006).

38. Zhou, Y. \& Ni, M. SHORT HYPOCOTYL UNDER BLUE1 truncations and mutations alter its association with a signaling protein complex in arabidopsis. Plant Cell 3, 703-715 (2010).

39. Yamashino, T. et al. A link between Circadian-controlled bHLH factors and the APRR1/TOC1 quintet in Arabidopsis thaliana. Plant Cell Physiol. 44, 619-629 (2003).

40. Nozue, K. et al. Rhythmic growth explained by coincidence between internal and external cues. Nature 448, 358-361 (2007).

41. Niwa, Y., Yamashino, T. \& Mizuno, T. The circadian clock regulates the photoperiodic response of hypocotyl elongation through a coincidence mechanism in Arabidopsis thaliana. Plant Cell Physiol. 50, 838-854 (2009).

42. Fernandez, V., Takahashi, Y., Le Gourrierec, J. \& Coupland, G. Photoperiodic and thermosensory pathways interact through CONSTANS to promote flowering at high temperature under short days. Plant J. 86, 426-440 (2016).

43. Dubos, C. et al. MYB transcription factors in Arabidopsis. Trends Plant Sci. 15, 573-581 (2010).

44. Zhou, Y. et al. SHORT HYPOCOTYL UNDER BLUE1 associates with MINISEED3 and HAIKU2 promoters in vivo to control Arabidopsis seed development. Plant Cell 21, 106-117 (2009).

45. O'Neill, J. P., Colon, K. T. \& Jenik, P. D. The onset of embryo maturation in Arabidopsis is determined by its developmental stage and does not depend on endosperm cellularization. Plant J. https://doi.org/10.1111/tpj.14324 (2019).

46. Nusinow, D. A. et al. The ELF4-ELF3-LUX complex links the circadian clock to diurnal control of hypocotyl growth. Nature 475, 398-402 (2011).

47. Nakamichi, N. et al. Transcriptional repressor PRR5 directly regulates clockoutput pathways. Proc. Natl Acad. Sci. USA. 109, 17123-17128 (2012).

48. Nagel, D. H. et al. Genome-wide identification of CCA1 targets uncovers an expanded clock network in Arabidopsis. Proc. Natl Acad. Sci. USA. 34, 4802-4810 (2015).

49. Lu, S. X., Knowles, S. M., Andronis, C., Ong, M. S. \& Tobin, E. M. CIRCADIAN CLOCK ASSOCIATED1 and LATE ELONGATED HYPOCOTYL function synergistically in the circadian clock of Arabidopsis. Plant Physiol. 150, 834-843 (2009).

50. Wang, Z. Y. et al. A Myb-related transcription factor is involved in the phytochrome regulation of an Arabidopsis Lhcb gene. Plant Cell 4, 491-507 (1997).

51. Quail, P. H. Photosensory perception and signalling in plant cells: new paradigms? Curr. Opin. Cell Biol. 14, 180-188 (2002).
52. Kidokoro, S. et al. The phytochrome-interacting factor PIF7 negatively regulates DREB1 expression under circadian control in Arabidopsis. Plant Physiol. 151, 2046-2057 (2009).

53. Huq, E. \& Quail, P. H. PIF4, a phytochrome-interacting bHLH factor, functions as a negative regulator of phytochrome Bsignaling in Arabidopsis. EMBO J. 21, 2441-2450 (2002).

54. Leivar, P. et al. The Arabidopsis phytochrome-interacting factor PIF7, together with PIF3 and PIF4, regulates responses to prolonged red light by modulating phyB levels. Plant Cell 20, 337-352 (2008).

55. Zhu, Y. et al. Phytochrome B binds with greater apparent affinity than phytochrome A to the basic helix-loop-helix factor PIF3 in a reaction requiring the PAS domain of PIF3. Proc. Natl Acad. Sci. USA. 97, 13419-13424 (2000).

56. Duek, P. D. \& Fankhauser, C. bHLH class transcription factors take center stage in phytochrome signalling. Trends Plant Sci. 10, 51-54 (2005).

57. Monte, E., Al-Sady, B., Leivar, P. \& Quail, P. H. Out of the dark: how the PIFs are unmasking a dual temporal mechanism of phytochrome signalling. J. Exp. Bot. 58, 3125-3133 (2007).

58. Khanna, R. et al. A novel molecular recognition motif necessary for targeting photoactivated phytochrome signaling to specific basic helix-loop-helix transcription factors. Plant Cell 16, 3033-3044 (2004).

59. Kumar, S. V. et al. Transcription factor PIF4 controls the thermosensory activation of flowering. Nature 484, 242-245 (2012).

60. Lorrain, S., Allen, T., Duek, P. D., Whitelam, G. C. \& Fankhauser, C. Phytochrome-mediated inhibition of shade avoidance involves degradation of growth-promoting bHLH transcription factors. Plant J. 53, 312-323 (2008).

61. Zhang, B. et al. BLADE-ON-PETIOLE proteins act in an E3 ubiquitin ligase complex to regulate PHYTOCHROME INTERACTING FACTOR 4 abundance. Elife 6, e26759 (2017).

62. Bowler, C. et al. Chromatin techniques for plant cells. Plant J. 39, 776-789 (2004).

63. Walter, M. et al. Visualization of protein interactions in living plant cells using bimolecular fluorescence complementation. Plant J. 40, 428-438 (2004).

64. Yoo, S. D., Cho, Y. H. \& Sheen, J. Arabidopsis mesophyll protoplasts: a versatile cell system for transient gene expression analysis. Nat. Protoc. 7, 1565-1575 (2007).

\section{Acknowledgements}

We thank Gang Li for 35 S::CCA1:FLAG, cca1-11, cca1-11 lhy-21, and 35 S::ELF4:GFP seeds and ABRC for $l$ hy- 21 seeds. This work was supported by a grant from National Natural Science Foundation of China (31470379) and a grant from the USDA National Institution of Food and Agriculture grant 2011-67013-30150.

\section{Author contributions}

M.N. and Q.S. conceived and designed the research plan. Q.S., S.W., G.X., X.K., and M.Z. performed the experiments. Q.S. and M.N. wrote the manuscript.

\section{Additional information}

Supplementary Information accompanies this paper at https://doi.org/10.1038/s41467019-11071-6.

Competing interests: The authors declare no competing interests.

Reprints and permission information is available online at http://npg.nature.com/ reprintsandpermissions/

Peer review information: Nature Communications thanks the anonymous reviewers for their contribution to the peer review of this work. Peer reviewer reports are available.

Publisher's note: Springer Nature remains neutral with regard to jurisdictional claims in published maps and institutional affiliations.

Open Access This article is licensed under a Creative Commons Attribution 4.0 International License, which permits use, sharing, adaptation, distribution and reproduction in any medium or format, as long as you give appropriate credit to the original author(s) and the source, provide a link to the Creative Commons license, and indicate if changes were made. The images or other third party material in this article are included in the article's Creative Commons license, unless indicated otherwise in a credit line to the material. If material is not included in the article's Creative Commons license and your intended use is not permitted by statutory regulation or exceeds the permitted use, you will need to obtain permission directly from the copyright holder. To view a copy of this license, visit http://creativecommons.org/ licenses/by/4.0/.

(C) The Author(s) 2019 